\title{
Quadratic derivative nonlinear Schrödinger equations in two space dimensions
}

\author{
Fernando Bernal-Vílchis, Nakao Hayashi and Pavel I. Naumkin
}

\begin{abstract}
We study the global in time existence of small classical solutions to the nonlinear Schrödinger equation with quadratic interactions of derivative type in two space dimensions

$$
\begin{cases}i \partial_{t} u+\frac{1}{2} \Delta u=\mathcal{N}(\nabla u, \nabla u), & t>0, x \in \mathbf{R}^{2}, \\ u(0, x)=u_{0}(x), & x \in \mathbf{R}^{2},\end{cases}
$$

where the quadratic nonlinearity has the form $\mathcal{N}(\nabla u, \nabla v)=\sum_{k, l=1,2} \lambda_{k l}$ $\left(\partial_{k} u\right)\left(\partial_{l} v\right)$ with $\lambda \in \mathbf{C}$. We prove that if the initial data $u_{0} \in \mathbf{H}^{6} \cap \mathbf{H}^{3,3}$ satisfy smallness conditions in the weighted Sobolev norm, then the solution of the Cauchy problem (0.1) exists globally in time. Furthermore we prove the existence of the usual scattering states. The proof depends on the energy type estimates, smoothing property by Doi, and method of normal forms by Shatah.
\end{abstract}

Mathematics Subject Classification (1991). Primary 35Q35.

Keywords. Nonlinear Schrödinger equations, Global existence, Quadratic nonlinearities, Two spatial dimensions.

\section{Introduction}

We consider the Cauchy problem for the quadratic derivative nonlinear Schr ödinger equation in two space dimensions

$$
\begin{cases}i \partial_{t} u+\frac{1}{2} \Delta u=\mathcal{N}(\nabla u, \nabla u), & t>1, \quad x \in \mathbf{R}^{2}, \\ u(1, x)=u_{0}(x), & x \in \mathbf{R}^{2},\end{cases}
$$

where the quadratic nonlinearity has the form $\mathcal{N}(\nabla u, \nabla v)=\sum_{k, l=1,2} \lambda_{k l}\left(\partial_{k} u\right)$ $\left(\partial_{l} v\right)$ with $\lambda \in \mathbf{C}$. Without a loss of a generality we can suppose that $\lambda$ is symmetric: $\lambda_{k l}=\lambda_{l k}$. For the convenience of the forthcoming calculations we take the initial time at $t=1$. The difficulty in the study of the large time behavior of solutions to the Cauchy problem (1.1) is that the quadratic nonlinearity in two space dimensions is critical since the nonlinearity of degree 2 may act 
as a long range perturbation in two space dimensions. The special oscillating structure of the nonlinearity must be taken into account to show that the solution has the usual scattering properties. Note that the structure of the quadratic nonlinearity under consideration is not gauge invariant that is it does not satisfy the property $\mathcal{N}\left(u e^{i \theta}\right)=e^{i \theta} \mathcal{N}(u)$ for all $\theta \in \mathbf{R}$. This fact does not allow us to estimate the operator $\mathcal{J}=x+i t \nabla$ via the standard energy type methods. Another problem is the so-called derivative loss difficulty.

There is a large literature on the Cauchy problem for Eq. (1.1) in the case of higher space dimensions (see, for instance $[1-4,10,14,16,20-24,26,28,30-$ $34,38,40],[36,37,43]$, and references therein). The classical energy method naturally requires that the real part of every component of $\frac{\partial F}{\partial(\nabla u)}$ vanishes that is

$$
\operatorname{Re} \frac{\partial F}{\partial(\nabla u)}=0 .
$$

With the condition $(1.2)$, in papers $[30,31,38]$, it was proved the global existence of classical solutions for small Cauchy data with sufficient regularity and decay at infinity in space dimension $n \geq 5$. Here the decay at infinity was imposed in such a way that $u_{0} \in \mathbf{H}_{p^{\prime}}^{m}\left(\mathbf{R}^{n}\right)$ with an integer $m>\frac{n}{2}, p>2$, where $\mathbf{H}_{p}^{m}\left(\mathbf{R}^{n}\right)$ is the Sobolev space and $1 / p+1 / p^{\prime}=1$. In particular, it was obtained an explicit time-decay of solutions in $\mathbf{L}^{p}\left(\mathbf{R}^{n}\right), 2 \leq p \leq 4$.

In papers $[3,4,20]$, condition (1.2) was removed by using the zeroth order pseudo-differential operator to get smoothing properties. In [3,4,20,29], decay at infinity was imposed in such a way that $x^{\alpha} u_{0} \in \mathbf{H}^{m}\left(\mathbf{R}^{n}\right)$ with an integer $m>\frac{n}{2},|\alpha| \leq 2$, which also provides an explicit time-decay of solutions in $\mathbf{L}^{p}\left(\mathbf{R}^{n}\right)$ for some $p>2$ through the first order partial differential operators having special commutator relations with $i \partial_{t}+\frac{1}{2} \Delta$.

In paper [35], it was removed the assumption on the decay at infinity of the Cauchy data $u_{0}$ and the regularity of $u_{0}$ was reduced such as $u_{0} \in \mathbf{H}^{s}\left(\mathbf{R}^{n}\right)$ with $s>\frac{n}{2}+2$. The condition $s>\frac{n}{2}+2$ is more natural in the framework of the classical solutions. Instead, there were some assumptions on the degree of the nonlinearity which are stronger than the cubic order of the nonlinearity and condition (1.2).

Global existence of small solutions to nonlinear Schrödinger equations with quadratic nonlinearities first was studied in papers $[30,31,38]$ under the condition (1.2) and the space dimension $n \geq 5$. Also in papers $[15,17,20]$ the case of the general nonlinearity was studied for the space dimension $n \geq 5$. In paper [13] the global existence was proved for the case $n=4$ with condition (1.2). The almost global existence for quadratic NLS was proved in paper [1] for space dimension $n=3$ under the conditions (1.2). (By an almost global existence of solutions we call the estimate $T=O\left(\exp \left(\frac{C}{\varepsilon}\right)\right)$ for the existence time $T$ of solutions, where $\varepsilon>0$ denotes the size of the initial data in a suitable norm.) Condition (1.2) was removed in [15], where the global existence of small solutions to the Cauchy problem (1.1) with general nonlinearity was proved for any space dimension $n \geq 5$. Note that the problem on the global existence of solutions become more difficult in the case of low space dimensions. In paper 
[20] the global existence was obtained for the case $n=3,4$ under the condition

$$
\left|\frac{\partial \mathcal{N}}{\partial u}\right|+\left|\frac{\partial \mathcal{N}}{\partial \bar{u}}\right| \leq C|\nabla u| \text {. }
$$

Global existence was proved in paper [20] for space dimensions $n=3,4$ under the condition (1.3), and in paper [12] for space dimensions $n=3,4$ under the conditions (1.2) and (1.3). The almost global existence for quadratic NLS was proved in paper [13] for space dimension $n=2$ under the conditions (1.2) and (1.3). Recently the nonlinear term

$$
\mathcal{N}=\lambda u^{2}+\mu \bar{u}^{2}
$$

where $\lambda, \mu \in \mathbf{C}$, was considered in [18] and the global existence of small solutions was shown in three space dimensions. However for the case of low space dimensions there are only few results. In the exceptional case

$$
\mathcal{N}=(\nabla u)^{2}
$$

Equation (1.1) can be linearized by the Hopf-Cole transformation and the solution can be written explicitly, so the global existence and the asymptotic properties of the solution can be studied, see paper [33]. The almost global existence was proved in [11] for the case $n=3$. In the case of two space dimensions the almost global existence of small analytic solutions to the Cauchy problem (1.1) with the nonlinearity of the form

$$
\mathcal{N}=\sum_{j, k=1}^{2}\left(\lambda_{j k}\left(\partial_{x_{j}} u\right)\left(\partial_{x_{k}} u\right)+\mu_{j k}\left(\partial_{x_{j}} \bar{u}\right)\left(\partial_{x_{k}} \bar{u}\right)+\nu_{j k}\left(\partial_{x_{j}} u\right)\left(\partial_{x_{k}} \bar{u}\right)\right)
$$

was studied in paper [17]. Moreover the global existence of small analytic solutions to the problem (1.1) was proved in the case of the nonlinearity

$$
\mathcal{N}=\lambda\left(\left(\partial_{x_{1}} u\right)\left(\partial_{x_{2}} \bar{u}\right)-\left(\partial_{x_{1}} \bar{u}\right)\left(\partial_{x_{2}} u\right)\right)
$$

where $\lambda \in \mathbf{C}$. In paper [6], the global existence of small solutions in the usual Sobolev spaces was established for the case of the nonlinearity

$$
\mathcal{N}=\sum_{j, k=1}^{2} \mu_{j k}\left(\partial_{x_{j}} \bar{u}\right)\left(\partial_{x_{k}} \bar{u}\right)
$$

for space dimension $n=2$ by using the method of the normal forms introduced in [39]. We note that the method used in [6] does not work for the case $\lambda_{j k} \neq 0$. The global existence for quadratic NLS was proved in paper [42] for space dimension $n=4$ under the conditions

$$
\mathcal{N} \text { does not depend on } \nabla u \text { and } \nabla \bar{u} \text {. }
$$

Also in paper [42] it was proved the almost global existence for quadratic NLS under the conditions (1.8) for space dimension $n=3$. Note that the nonlinearity (1.4) is a particular case of (1.8). Recently in paper [9] it was proved the global existence of solutions to the quadratic nonlinear Schrödinger equations of the form (1.8) in 3 spatial dimensions. The global existence for quadratic 
NLS was proved in paper $[15,17,20]$ for space dimension $n=3$ under the condition

$$
\mathcal{N} \text { does not depend on } u \text { and } \bar{u} \text {. }
$$

The nonlinearity (1.6) and the Hopf-Cole case (1.5) are also the consequences of (1.9). The almost global existence for quadratic NLS was proved in paper [17] for space dimension $n=2$ under the condition (1.9). The estimate $T=O\left(\varepsilon^{-6}\right)$ for the time existence of solutions was found in paper [5] by using the method of normal forms if the nonlinearity $\mathcal{N}=\left(\bar{u}_{x}\right)^{2}$ in one spatial dimension. The global existence of small analytic solutions to the Cauchy problem (1.1) in the case of two space dimensions with the nonlinearity

$$
\mathcal{N}=\sum_{j, k=1}^{2}\left(\lambda_{j k}\left(\partial_{x_{j}} u\right)\left(\partial_{x_{k}} u\right)+\mu_{j k}\left(\partial_{x_{j}} \bar{u}\right)\left(\partial_{x_{k}} \bar{u}\right)\right)
$$

was studied in paper [19]. In paper [7] it was proved the global existence of solutions to the quadratic nonlinear Schrödinger equation in two dimensional case with the nonlinearity which do depends on the derivatives of $u$ when the initial data $u_{0} \in \mathbf{H}^{10,0}\left(\mathbf{R}^{2}\right) \cap \mathbf{H}^{0,10}\left(\mathbf{R}^{2}\right)$ are small. To overcome the long-range difficulty it was applied a method similar to the normal forms of Shatah [39]. To remove the derivative loss problem the local smoothing property of Kato in the version of paper [28] was used.

In the present paper we improve the regularity conditions on the initial data such as $u_{0} \in \mathbf{H}^{6}\left(\mathbf{R}^{2}\right) \cap \mathbf{H}^{3,3}\left(\mathbf{R}^{2}\right)$ comparing with paper [7]. We overcome the long-range difficulty using a version of the normal forms of Shatah [39]. To overcome the problem of the derivatives loss we follow the Doi's method of the zeroth order pseudo-differential operator for getting the smoothing properties [8]. The last method is much more simple and allow us to generalize the regularity conditions on the initial data. The important fact is that the nonlinearity under consideration has the type (1.9), which helps us to evaluate the large time asymptotics of the nonlinearity, since the remainder terms can be estimated via the operator $\mathcal{I}=x \cdot \nabla+2 t \partial_{t}$ instead of the operator $\mathcal{J} \cdot \nabla$, where the vector $\mathcal{J}=x+i t \nabla$. Also we define $\Omega=x_{1} \partial_{2}-x_{2} \partial_{1}$ which acts as the angular derivative. The operators $\mathcal{J}$ and $\Omega$ are useful to obtain the time decay properties of the linear Schrödinger evolution group

$$
\mathcal{U}(t)=\mathcal{F}^{-1} e^{-\frac{i t}{2} \xi^{2}} \mathcal{F}=i M(t) \mathcal{D}(t) \mathcal{F} M(t),
$$

where $M(t)=e^{\frac{i|x|^{2}}{2 t}}$, the dilation operator is $(\mathcal{D}(t) \varphi)(x)=\frac{1}{t} \varphi\left(\frac{x}{t}\right)$, where $\mathcal{F} \phi \equiv$ $\hat{\phi}(\xi)=\frac{1}{2 \pi} \int_{\mathbf{R}^{2}} e^{-i(x \cdot \xi)} \phi(x) d x$ denotes the Fourier transform of the function $\phi(x)$, and $\mathcal{F}^{-1}$ is the inverse Fourier transformation defined by $\mathcal{F}^{-1} \phi=$ $\frac{1}{2 \pi} \int_{\mathbf{R}^{2}} e^{i(x \cdot \xi)} \phi(\xi) d \xi$. The inverse free Schrödinger evolution group is written as $\mathcal{U}(-t)=-\bar{M}(t) i \mathcal{F}^{-1} \mathcal{D}\left(\frac{1}{t}\right) \bar{M}(t)$, where $\mathcal{D}^{-1}(t)=-i \mathcal{D}\left(\frac{1}{t}\right)$ is the inverse dilation operator. We use freely in the paper the following commutation relations $[\mathcal{I}, \mathcal{J}]=\mathcal{J},[\mathcal{I}, \nabla]=-\nabla,[\Omega, \mathcal{I}]=[\Omega, \mathcal{L}]=[\nabla, \mathcal{L}]=0,[\mathcal{I}, \mathcal{L}]=2 \mathcal{L},\left[\mathcal{L}, \frac{x}{t}\right]=$ $-i t^{-2} \mathcal{J},\left[x_{k}, \nabla_{j}\right]=i \delta_{k j},\left[\Omega, \partial_{l}\right]=\delta_{2 l} \partial_{1}-\delta_{1 l} \partial_{2}$, where $\delta_{j k}=1$ if $j=k$ and $\delta_{j k}=0$ if $j \neq k$. 
To state our result precisely, we now give some notations. We denote the usual Lebesgue space by $\mathbf{L}^{p}=\mathbf{L}^{p}\left(\mathbf{R}^{2}\right)$ with the norm $\|\phi\|_{\mathbf{L}^{p}}=$ $\left(\int_{\mathbf{R}^{2}}|\phi(x)|^{p} d x\right)^{1 / p}$ if $1 \leq p<\infty$ and $\|\phi\|_{\mathbf{L}^{\infty}}=$ ess. $\sup \left\{|\phi(x)| ; x \in \mathbf{R}^{2}\right\}$. The weighted Sobolev space is defined by $\mathbf{W}_{p}^{m, k}=\left\{\phi \in \mathbf{L}^{2}:\left\|\langle x\rangle^{k}\langle i \nabla\rangle^{m} \phi\right\|_{\mathbf{L}^{p}}<\infty\right\}$, where $m, k \in \mathbf{R}^{+}, 1 \leq p \leq \infty,\langle x\rangle=\sqrt{1+x^{2}}$. For simplicity we write $\mathbf{H}^{m, s}=\mathbf{W}_{2}^{m, s}, \mathbf{H}^{m}=\mathbf{W}_{2}^{m}, \mathbf{W}_{p}^{m}=\mathbf{W}_{p}^{m, 0}$ and $\mathbf{L}^{p, k}=\mathbf{H}_{p}^{0, k}$. The index 0 we usually omit if it does not cause a confusion. Let $\mathbf{C}(\mathbf{I} ; \mathbf{B})$ be the space of continuous functions from a time interval $\mathbf{I}$ to a Banach space B. Different positive constants we denote by the same letter $C$.

Now we state our result.

Theorem 1.1. Let the initial data $u_{0} \in \mathbf{H}^{6} \cap \mathbf{H}^{3,3}$ have a sufficiently small norm $\left\|u_{0}\right\|_{\mathbf{H}^{6}}+\left\|u_{0}\right\|_{\mathbf{H}^{3,3}}$. Then there exists a unique global solution $u \in \mathbf{C}\left(\mathbf{R}, \mathbf{H}^{6} \cap\right.$ $\mathbf{H}^{3,3}$ ) to the Cauchy problem (1.1). Moreover there exists a unique final state $u_{+} \in \mathbf{L}^{2}$ such that

$$
\left\|\mathcal{U}(-t) \nabla u(t)-u_{+}\right\|_{\mathbf{L}^{2}} \leq C t^{-\omega}
$$

as $t \rightarrow \infty$, where $0<\omega<\frac{1}{3}$.

Remark 1.1. Similar result is true for the case of the negative time.

For the convenience of the reader we now explain our strategy of the proof. Analogously to the Shatah normal forms [39] we now transform the nonlinear term in the right-hand side of (1.1). Note that the identity is true

$$
\mathcal{L}(A v w)=v w \mathcal{L} A+A(w \mathcal{L} v+v \mathcal{L} w)+A \nabla v \cdot \nabla w+\nabla A \cdot \nabla(v w)
$$

with the operator $\mathcal{L}=i \partial_{t}+\frac{1}{2} \Delta$. Using the definition of the operator $\mathcal{J}=$ $x+i t \nabla$, we can express the derivative $\nabla$ through an explicit time decay $\nabla=$ $i t^{-1}(x-\mathcal{J})$. Therefore we obtain the following identity

$$
\begin{aligned}
\mathcal{L}(A v w)= & v w\left(-t^{-2}|x|^{2} A+\mathcal{L} A+2 i t^{-1}(x \cdot \nabla) A\right) \\
& +A(w \mathcal{L} v+v \mathcal{L} w)+t^{-2} v b \cdot \mathcal{J} w+t^{-2} w b \cdot \mathcal{J} v \\
& -\frac{i}{2 t} A(\nabla v \cdot \mathcal{J} w+\nabla w \cdot \mathcal{J} v),
\end{aligned}
$$

where $b=\frac{1}{2} A x-i t \nabla A$. Denote $B=\left\langle\frac{x}{\sqrt{t}}\right\rangle^{-1}$. Taking $A=\lambda_{k l} t B^{2}, v=\partial_{k} u$, $w=\partial_{l} u$ in identity (1.11) and using Eq. (1.1) we get for the new unknown function $h=u+t B^{2} \mathcal{N}(\nabla u, \nabla u)$ the following equation

$$
\begin{aligned}
\mathcal{L} h= & a \mathcal{N}(\nabla u, \nabla u)+2 t B^{2} \mathcal{N}(\nabla u, \nabla \mathcal{L} u) \\
& +\sum_{j=1,2} b_{j} \mathcal{N}\left(\nabla u, \mathcal{J}_{j} \nabla u\right)-\sum_{j=1,2} i B^{2} \mathcal{N}\left(\partial_{j} \nabla u, \mathcal{J}_{j} \nabla u\right),
\end{aligned}
$$

where $a=B^{2}+\mathcal{L}\left(t B^{2}\right)+2 i(x \cdot \nabla) B^{2}$ and $b_{j}=t^{-1} x_{j} B^{2}-2 i\left(\partial_{j} B^{2}\right)$. By a direct calculation we see that $\left|\nabla B^{2}\right| \leq C t^{-\frac{1}{2}} B^{3},\left|\mathcal{L}\left(t B^{2}\right)\right| \leq C B^{2}$ and $|a| \leq C B^{2}$, $\left|b_{j}\right| \leq C t^{-\frac{1}{2}} B$. All the terms of the right-hand side of equation (1.12) contain the decaying function $B=\left\langle\frac{x}{\sqrt{t}}\right\rangle^{-1}$, so that the action of the operator $\mathcal{J}=x+i t \nabla$ can be estimated easily. Also the presence of the spacial derivative and the function $B$ improve the time decay property, for example, by the 
relation $B \nabla u=i t^{-1} x B u-i t^{-1} B \mathcal{J} u$ we can see that $B \nabla u$ has a better decay property than $u$ (see Lemma 3.2 below).

The rest of the paper we organize as follows. In Sect. 2 we state the smoothing property of Doi [8] and give some preliminary estimates. In Sect. 3 we give estimates for the solutions of Eq. (1.1) in our basic norm

$$
\|u\|_{\mathbf{X}_{T}}=\sup _{t \in[1, T]}\left(t^{-\gamma}\left\|\Gamma^{3} u\right\|_{\mathbf{L}^{2}}+t^{-3 \gamma}\left\|\mathcal{J} \Gamma^{2} u\right\|_{\mathbf{L}^{2}}+\left\|\Gamma^{2} u\right\|_{\mathbf{L}^{2}}\right),
$$

where $\Gamma=(1, \mathcal{I}, \Omega, \Delta), \mathcal{I}=(x \cdot \nabla)-2 t \partial_{t}$ and $\Omega=x_{2} \partial_{1}-x_{1} \partial_{2}, \gamma>0$ is small. Section 4 is devoted to the proof of Theorem 1.1.

\section{Smoothing property}

The aim of this section is to obtain the smoothing effect for solutions to the Cauchy problem for the linear Schrödinger equation

$$
\begin{cases}\mathcal{L} u=f, & x \in \mathbf{R}^{n}, \quad t>1 \\ u(1, x)=u_{0}(x), & x \in \mathbf{R}^{n},\end{cases}
$$

where the function $f$ is a given force, the space dimension $n \geq 1$. Smoothing effects for solutions to the Cauchy problem (2.1) were studied by many authors (see $[8,27]$ and references therein). In our key Lemma 2.2 of this section we will obtain a simple and explicit modification of smoothing effects of Doi [8]. We choose the following zeroth order pseudo-differential operator

$$
\mathcal{S}=\prod_{j=1}^{n}\left(\cosh \left(\chi\left(\frac{x_{j}}{t}\right)\right)+i \sinh \left(\chi\left(\frac{x_{j}}{t}\right)\right) \mathcal{H}_{j}\right),
$$

where $\chi(x)=\arctan x$, and

$$
\mathcal{H}_{j} \phi=\frac{1}{\pi} \mathrm{PV} \int_{\mathbf{R}} \phi\left(x-\tau \mathbf{1}_{j}\right) \frac{d \tau}{\tau}
$$

is the Hilbert transformation with respect to the coordinate $x_{j}, \mathrm{PV}$ means the principal value of the singular integral and $\mathbf{1}_{j}=\left(\delta_{k, j}\right)_{(k=1, \ldots, n)}$, with $\delta_{k, j}=1$ if $j=k$ and $\delta_{k, j}=0$ if $j \neq k$ is the Kronecker delta symbol. We also note that the operator $\mathcal{S}$ acts continuously from $\mathbf{L}^{2}$ to $\mathbf{L}^{2}$ with the following estimate $\|\mathcal{S} \phi\|_{\mathbf{L}^{2}} \leq C\|\phi\|_{\mathbf{L}^{2}}$. The inverse operator

$$
\mathcal{S}^{-1}=\prod_{j=1}^{n}\left(1+i \tanh \left(\chi\left(\frac{x_{j}}{t}\right)\right) \mathcal{H}_{j}\right)^{-1} \frac{1}{\cosh \left(\chi\left(\frac{x_{j}}{t}\right)\right)}
$$

exists and is continuous $\left\|\mathcal{S}^{-1} \phi\right\|_{\mathbf{L}^{2}} \leq C\|\phi\|_{\mathbf{L}^{2}}$. We denote the fractional partial derivative $\left|\partial_{j}\right|^{\alpha}$ for $\alpha \in(0,1), j=1,2, \ldots, n$, as follows (see [41])

$$
\left|\partial_{j}\right|^{\alpha} \phi(x)=\mathcal{F}^{-1}\left(\left|x_{j}\right|^{\alpha} \widehat{\phi}(x)\right)=C \int_{\mathbf{R}}\left(\phi\left(x-\tau \mathbf{1}_{j}\right)-\phi(x)\right) \frac{d \tau}{|\tau|^{1+\alpha}}
$$

and similarly

$$
\left|\partial_{j}\right|^{\alpha} \mathcal{H}_{j} \phi=\mathcal{F}^{-1}\left(i \operatorname{sign} x_{j}\left|x_{j}\right|^{\alpha} \widehat{\phi}(x)\right)=C \int_{\mathbf{R}}\left(\phi\left(x-\tau \mathbf{1}_{j}\right)-\phi(x)\right) \frac{d \tau}{\tau|\tau|^{\alpha}} .
$$


We can write the Leibnitz rule as follows $\left|\partial_{j}\right|^{\alpha}(\phi \psi)=\phi\left|\partial_{j}\right|^{\alpha} \psi+$ $\left[\left|\partial_{j}\right|^{\alpha}, \phi\right] \psi$, where the commutator

$$
\left[\left|\partial_{j}\right|^{\alpha}, \phi\right] \psi=C \int_{\mathbf{R}}\left(\phi\left(x-\tau \mathbf{1}_{j}\right)-\phi(x)\right) \psi\left(x-\tau \mathbf{1}_{j}\right) \frac{d \tau}{|\tau|^{1+\alpha}} .
$$

In the next lemma we find the estimates for the $\mathbf{L}^{p}$-norms of the commutators $\left[\left|\partial_{j}\right|^{\alpha}, \phi\right] \psi$ and

$$
\left[\left|\partial_{j}\right|^{\alpha} \mathcal{H}_{j}, \phi\right] \psi=C \int_{\mathbf{R}}\left(\phi\left(x-\tau \mathbf{1}_{j}\right)-\phi(x)\right) \psi\left(x-\tau \mathbf{1}_{j}\right) \frac{d \tau}{\tau|\tau|^{\alpha}}
$$

Lemma 2.1. The inequalities are valid

$$
\left\|\left[\left|\partial_{j}\right|^{\alpha}, \phi\right] \psi\right\|_{\mathbf{L}^{p}}+\left\|\left[\left|\partial_{j}\right|^{\alpha} \mathcal{H}_{j}, \phi\right] \psi\right\|_{\mathbf{L}^{p}} \leq C\left\|\partial_{j} \phi\right\|_{\mathbf{L}^{q}}^{\alpha}\|\phi\|_{\mathbf{L}^{q}}^{1-\alpha}\|\psi\|_{\mathbf{L}^{r}}
$$

where $0<\alpha<1, \frac{1}{p}=\frac{1}{q}+\frac{1}{r}$, provided that the right-hand side is finite.

Proof. We have

$$
\begin{aligned}
\left\|\phi\left(x-\tau \mathbf{1}_{j}\right)-\phi(x)\right\|_{\mathbf{L}^{q}} & \leq C\left\|\int_{0}^{\tau} \partial_{j} \phi\left(x-z \mathbf{1}_{j}\right) d z\right\|_{\mathbf{L}^{q}} \\
& \leq C \int_{0}^{\tau}\left\|\partial_{j} \phi\right\|_{\mathbf{L}^{q}} d z \leq C|\tau|\left\|\partial_{j} \phi\right\|_{\mathbf{L}^{q}}
\end{aligned}
$$

for $1 \leq q \leq \infty$. Therefore choosing $\delta=\left\|\partial_{j} \phi\right\|_{\mathbf{L}^{q}}^{-1}\|\phi\|_{\mathbf{L}^{q}}>0$ we get by the Hölder inequality

$$
\begin{aligned}
\left\|\left[\left|\partial_{j}\right|^{\alpha}, \phi\right] \psi\right\|_{\mathbf{L}^{p}} & =C\left\|\int_{\mathbf{R}}\left(\phi\left(x-\tau \mathbf{1}_{j}\right)-\phi(x)\right) \psi\left(x-\tau \mathbf{1}_{j}\right) \frac{d \tau}{|\tau|^{1+\alpha}}\right\|_{\mathbf{L}^{p}} \\
& \leq C \int_{\mathbf{R}}\left\|\left(\phi\left(x-\tau \mathbf{1}_{j}\right)-\phi(x)\right) \psi\left(x-\tau \mathbf{1}_{j}\right)\right\|_{\mathbf{L}^{p}} \frac{d \tau}{|\tau|^{1+\alpha}} \\
& \leq C\|\psi\|_{\mathbf{L}^{r}} \int_{\mathbf{R}}\left\|\phi\left(x-\tau \mathbf{1}_{j}\right)-\phi(x)\right\|_{\mathbf{L}^{q}} \frac{d \tau}{|\tau|^{1+\alpha}} \\
& \leq C\left(\left\|\partial_{j} \phi\right\|_{\mathbf{L}^{q}} \int_{|\tau| \leq \delta} \frac{d \tau}{|\tau|^{\alpha}}+\|\phi\|_{\mathbf{L}^{q}} \int_{|\tau|>\delta} \frac{d \tau}{|\tau|^{1+\alpha}}\right)\|\psi\|_{\mathbf{L}^{r}} \\
& \leq C\left(\delta^{1-\alpha}\left\|\partial_{j} \phi\right\|_{\mathbf{L}^{q}}+\delta^{-\alpha}\|\phi\|_{\mathbf{L}^{q}}\right)\|\psi\|_{\mathbf{L}^{r}} \leq C\left\|\partial_{j} \phi\right\|_{\mathbf{L}^{q}}^{\alpha}\|\phi\|_{\mathbf{L}^{q}}^{1-\alpha}\|\psi\|_{\mathbf{L}^{r}} .
\end{aligned}
$$

In the same manner we estimate the norm $\left\|\left[\left|\partial_{j}\right|^{\alpha} \mathcal{H}_{j}, \phi\right] \psi\right\|_{\mathbf{L}^{p}}$. Lemma 2.1 is proved.

Operator $\mathcal{S}$ helps us to obtain the smoothing property for the linear Schrödinger equation by virtue of the usual energy type estimates. In the next lemma we prepare a generalized energy estimate, involving the operator $\mathcal{S}$ in which we have an additional positive term giving us the gain of regularity of the half-derivative of the unknown function $u$. The following lemma follows from lemma in paper. We will give the proof for the convenience of the reader.

Lemma 2.2. The following inequality is valid

$$
\frac{d}{d t}\|\mathcal{S} u\|_{\mathbf{L}^{2}}^{2}+2 t^{-1} \sum_{j=1}^{n}\left\|\left\langle\frac{x_{j}}{t}\right\rangle^{-1} \mathcal{S}\left|\partial_{j}\right|^{\frac{1}{2}} u\right\|_{\mathbf{L}^{2}}^{2} \leq C t^{-2}\|u\|_{\mathbf{L}^{2}}^{2}+2|(\mathcal{S} f, \mathcal{S} u)|
$$

for all $t>1$, for the solutions $u$ of the linear Schrödinger equation (2.1). 
Proof. Applying the operator $\mathcal{S}$ to both sides of the linear Schrö dinger equation (2.1) we get

$$
\mathcal{L} \mathcal{S} u-\frac{1}{2}[\Delta, \mathcal{S}] u-i\left[\partial_{t}, \mathcal{S}\right] u=\mathcal{S} f
$$

Since $\mathcal{H}_{j}^{2}=-1,\left|\partial_{j}\right|=-\partial_{j} \mathcal{H}_{j}$ and $\left[\partial_{j}, \mathcal{S}\right]=i\left(\partial_{j} \chi\left(\frac{x_{j}}{t}\right)\right) \mathcal{S} \mathcal{H}_{j}$, by the Leibnitz rule we obtain

$$
\begin{aligned}
{[\Delta, \mathcal{S}] u=} & \sum_{j=1}^{n}\left(\partial_{j} \chi\left(\frac{x_{j}}{t}\right)\right)^{2} \mathcal{S} u+i \sum_{j=1}^{n}\left(\partial_{j}^{2} \chi\left(\frac{x_{j}}{t}\right)\right) \mathcal{S H}_{j} u \\
& -2 \sum_{j=1}^{n} i\left(\partial_{j} \chi\left(\frac{x_{j}}{t}\right)\right) \mathcal{S}\left|\partial_{j}\right| u .
\end{aligned}
$$

Hence

$$
\begin{aligned}
\mathcal{L} \mathcal{S} u & +i \sum_{j=1}^{n}\left(\partial_{j} \chi\left(\frac{x_{j}}{t}\right)\right) \mathcal{S}\left|\partial_{j}\right| u=\sum_{j=1}^{n}\left(\frac{1}{2}\left(\partial_{j} \chi\left(\frac{x_{j}}{t}\right)\right)^{2} \mathcal{S} u\right. \\
+ & \left.\left(\frac{i}{2}\left(\partial_{j}^{2} \chi\left(\frac{x_{j}}{t}\right)\right)-\partial_{t} \chi\left(\frac{x_{j}}{t}\right)\right) \mathcal{S H}_{j} u\right)+\mathcal{S f} .
\end{aligned}
$$

Since $\partial_{j} \chi\left(\frac{x_{j}}{t}\right)=t^{-1}\left\langle\frac{x_{j}}{t}\right\rangle^{-2}, \partial_{t} \chi\left(\frac{x_{j}}{t}\right)=-\frac{x_{j}}{t^{2}}\left\langle\frac{x_{j}}{t}\right\rangle^{-2}$ we find

$$
\begin{aligned}
& \left|\left(\mathcal{S} u,\left(\frac{1}{2}\left(\partial_{j} \chi\left(\frac{x_{j}}{t}\right)\right)^{2} \mathcal{S} u+\left(\frac{i}{2}\left(\partial_{j}^{2} \chi\left(\frac{x_{j}}{t}\right)\right)-\partial_{t} \chi\left(\frac{x_{j}}{t}\right)\right) \mathcal{S H}_{j} u\right)\right)\right| \\
& \quad \leq C t^{-2}\|u\|_{\mathbf{L}^{2}}^{2} .
\end{aligned}
$$

Therefore multiplying both sides of Eq. (2.2) by $\overline{\mathcal{S} v}$, integrating over $\mathbf{R}^{n}$ and taking the imaginary part of the result, we get

$$
\begin{aligned}
& \frac{d}{d t}\|\mathcal{S} u\|_{\mathbf{L}^{2}}^{2}+2 t^{-1} \operatorname{Re} \sum_{j=1}^{n}\left(\left\langle\frac{x_{j}}{t}\right\rangle^{-2} \mathcal{S}\left|\partial_{j}\right| u, \mathcal{S} u\right) \\
& \quad \leq C t^{-2}\|u\|_{\mathbf{L}^{2}}^{2}+2|\operatorname{Im}(\mathcal{S} f, \mathcal{S} u)| .
\end{aligned}
$$

We next use the representation

$$
\begin{aligned}
& {\left[\left|\partial_{j}\right|^{\frac{1}{2}},\left\langle\frac{x_{j}}{t}\right\rangle^{-1} \mathcal{S}\right]=\left[\left|\partial_{j}\right|^{\frac{1}{2}},\left\langle\frac{x_{j}}{t}\right\rangle^{-1} \cosh \left(\chi\left(\frac{x_{j}}{t}\right)\right)\right] \prod_{k=1, k \neq j}^{n} \mathcal{S}_{k}} \\
& \quad+i\left[\left|\partial_{j}\right|^{\frac{1}{2}},\left\langle\frac{x_{j}}{t}\right\rangle^{-1} \sinh \left(\chi\left(\frac{x_{j}}{t}\right)\right)\right] \mathcal{H}_{j} \prod_{k=1, k \neq j}^{n} \mathcal{S}_{k},
\end{aligned}
$$


then by Lemma 2.1 we find

$$
\begin{aligned}
& \left\|\left[\left|\partial_{j}\right|^{\frac{1}{2}},\left\langle\frac{x_{j}}{t}\right\rangle^{-1} \mathcal{S}\right] \psi\right\|_{\mathbf{L}^{2}}+\left\|\left[\left|\partial_{j}\right|^{\frac{1}{2}} \mathcal{H}_{j},\left\langle\frac{x_{j}}{t}\right\rangle^{-1} \mathcal{S}\right] \psi\right\|_{\mathbf{L}^{2}} \\
& \leq C\left\|\partial_{j}\left(\left\langle\frac{x_{j}}{t}\right\rangle^{-1} \cosh \left(\chi\left(\frac{x_{j}}{t}\right)\right)\right)\right\|_{\mathbf{L}^{\infty}}^{\frac{1}{2}}\left\|\left\langle\frac{x_{j}}{t}\right\rangle^{-1} \cosh \left(\chi\left(\frac{x_{j}}{t}\right)\right)\right\|_{\mathbf{L}^{\infty}}^{\frac{1}{2}}\|\psi\|_{\mathbf{L}^{2}} \\
& \quad+C\left\|\partial_{j}\left(\left\langle\frac{x_{j}}{t}\right\rangle^{-1} \sinh \left(\chi\left(\frac{x_{j}}{t}\right)\right)\right)\right\|_{\mathbf{L}^{\infty}}^{\frac{1}{2}}\left\|\left\langle\frac{x_{j}}{t}\right\rangle^{-1} \sinh \left(\chi\left(\frac{x_{j}}{t}\right)\right)\right\|_{\mathbf{L}^{\infty}}^{\frac{1}{2}}\left\|\mathcal{H}_{j} \psi\right\|_{\mathbf{L}^{2}} \\
& \leq C t^{-\frac{1}{2}}\|\psi\|_{\mathbf{L}^{2}} .
\end{aligned}
$$

Hence we obtain

$$
\begin{aligned}
- & \operatorname{Re}\left(\left\langle\frac{x_{j}}{t}\right\rangle^{-2} \mathcal{S}\left|\partial_{j}\right| u, \mathcal{S} u\right) \\
= & \operatorname{Re}\left(\left\langle\frac{x_{j}}{t}\right\rangle^{-1} \mathcal{S} u,\left(\partial_{j}\left\langle\frac{x_{j}}{t}\right\rangle^{-1} \mathcal{S}+\left[\partial_{j},\left\langle\frac{x_{j}}{t}\right\rangle^{-1} \mathcal{S}\right]\right) \mathcal{H}_{j} u\right) \\
\leq & \operatorname{Re}\left(\left\langle\frac{x_{j}}{t}\right\rangle^{-1} \mathcal{S} u, \partial_{j}\left\langle\frac{x_{j}}{t}\right\rangle^{-1} \mathcal{S} \mathcal{H}_{j} u\right)+C t^{-1}\|u\|_{\mathbf{L}^{2}}^{2} \\
\leq & \operatorname{Re}\left(\left|\partial_{j}\right|^{\frac{1}{2}}\left\langle\frac{x_{j}}{t}\right\rangle^{-1} \mathcal{S} u,\left|\partial_{j}\right|^{\frac{1}{2}} \mathcal{H}_{j}\left\langle\frac{x_{j}}{t}\right\rangle^{-1} \mathcal{S} \mathcal{H}_{j} u\right)+C t^{-1}\|u\|_{\mathbf{L}^{2}}^{2} \\
\leq & \operatorname{Re}\left(\left\langle\frac{x_{j}}{t}\right\rangle^{-1} \mathcal{S}\left|\partial_{j}\right|^{\frac{1}{2}} u+\left[\left|\partial_{j}\right|^{\frac{1}{2}},\left\langle\frac{x_{j}}{t}\right\rangle^{-1} \mathcal{S}\right] u,\right. \\
& \left.\times\left\langle\frac{x_{j}}{t}\right\rangle^{-1} \mathcal{S}\left|\partial_{j}\right|^{\frac{1}{2}} u+\left[\left|\partial_{j}\right|^{\frac{1}{2}} \mathcal{H}_{j},\left\langle\frac{x_{j}}{t}\right\rangle^{-1} \mathcal{S}\right] \mathcal{H}_{j} u\right) \\
& +C t^{-1}\|u\|_{\mathbf{L}^{2}}^{2} \leq\left\|\left\langle\frac{x_{j}}{t}\right\rangle^{-1} \mathcal{S}\left|\partial_{j}\right|^{\frac{1}{2}} u\right\|_{\mathbf{L}^{2}}^{2}+C t^{-1}\|u\|_{\mathbf{L}^{2}}^{2} .
\end{aligned}
$$

Therefore by (2.3) and (2.4) the estimate of the lemma follows. Lemma 2.2 is proved.

The next lemma will be used to estimate the worst term of the nonlinearity with a derivative loss (see Lemma below).

Lemma 2.3. The estimate is true

$$
\begin{aligned}
\left|\left(\mathcal{S} u, \mathcal{S} \phi \partial_{j} u\right)\right| \leq & C\left(\left\|\left\langle\frac{x}{t}\right\rangle^{2} \phi\right\|_{\mathbf{L}^{\infty}}+\left\|\left\langle\frac{x}{t}\right\rangle \phi\right\|_{\mathbf{W}_{\infty}^{1}}\right) \\
& \times\left(\left\|\left\langle\frac{x_{j}}{t}\right\rangle^{-1} \mathcal{S}\left|\partial_{j}\right|^{\frac{1}{2}} u\right\|_{\mathbf{L}^{2}}^{2}+\|u\|_{\mathbf{L}^{2}}^{2}\right)
\end{aligned}
$$

provided that the right-hand side is bounded. 
Proof. We have the estimate

$$
\begin{aligned}
& \left|\left(\mathcal{S} u, \mathcal{S} \phi \partial_{j} u\right)\right|=\left|\left(\mathcal{S} u, \mathcal{S}\left\langle\frac{x_{j}}{t}\right\rangle \phi\left\langle\frac{x_{j}}{t}\right\rangle^{-1} \partial_{j} u\right)\right| \\
& \leq\left|\left(\mathcal{S} u, \mathcal{S}\left\langle\frac{x_{j}}{t}\right\rangle \phi\left|\partial_{j}\right|^{\frac{1}{2}} \mathcal{H}_{j}\left|\partial_{j}\right|^{\frac{1}{2}}\left\langle\frac{x_{j}}{t}\right\rangle^{-1} u\right)\right| \\
& \quad+\left|\left(\mathcal{S} u, \mathcal{S}\left\langle\frac{x_{j}}{t}\right\rangle \phi\left(\partial_{j}\left\langle\frac{x_{j}}{t}\right\rangle^{-1}\right) u\right)\right| \\
& \leq\left\|\left|\partial_{j}\right|^{\frac{1}{2}}\left\langle\frac{x_{j}}{t}\right\rangle \overline{\phi \mathcal{S}} \mathcal{S} u\right\|_{\mathbf{L}^{2}}\left\|\mathcal{H}_{j}\left|\partial_{j}\right|^{\frac{1}{2}}\left\langle\frac{x_{j}}{t}\right\rangle^{-1} u\right\|_{\mathbf{L}^{2}} \\
& \quad+C t^{-1}\|\phi\|_{\mathbf{L}^{\infty}}\|u\|_{\mathbf{L}^{2}}^{2} .
\end{aligned}
$$

By Lemma 2.1 we get

$$
\begin{aligned}
\left\|\left[\mathcal{S}\left|\partial_{j}\right|^{\frac{1}{2}},\left\langle\frac{x_{j}}{t}\right\rangle^{-1}\right] u\right\|_{\mathbf{L}^{2}} & \leq C\left\|\partial_{j}\left\langle\frac{x_{j}}{t}\right\rangle^{-1}\right\|_{\mathbf{L}^{\infty}}^{\frac{1}{2}}\left\|\left\langle\frac{x_{j}}{t}\right\rangle^{-1}\right\|_{\mathbf{L}^{\infty}}^{\frac{1}{2}}\|u\|_{\mathbf{L}^{2}} \\
& \leq C t^{-\frac{1}{2}}\|u\|_{\mathbf{L}^{2}} .
\end{aligned}
$$

Hence

$$
\begin{aligned}
& \left\|\mathcal{H}_{j}\left|\partial_{j}\right|^{\frac{1}{2}}\left\langle\frac{x_{j}}{t}\right\rangle^{-1} u\right\|_{\mathbf{L}^{2}}=\left\|\mathcal{S}^{-1} \mathcal{S}\left|\partial_{j}\right|^{\frac{1}{2}}\left\langle\frac{x_{j}}{t}\right\rangle^{-1} u\right\|_{\mathbf{L}^{2}} \\
& \leq C\left\|\mathcal{S}\left|\partial_{j}\right|^{\frac{1}{2}}\left\langle\frac{x_{j}}{t}\right\rangle^{-1} u\right\|_{\mathbf{L}^{2}} \\
& \leq\left\|\left\langle\frac{x_{j}}{t}\right\rangle^{-1} \mathcal{S}\left|\partial_{j}\right|^{\frac{1}{2}} u\right\|_{\mathbf{L}^{2}}+\left\|\left[\mathcal{S}\left|\partial_{j}\right|^{\frac{1}{2}},\left\langle\frac{x_{j}}{t}\right\rangle^{-1}\right] u\right\|_{\mathbf{L}^{2}} \\
& \leq\left\|\left\langle\frac{x_{j}}{t}\right\rangle^{-1} \mathcal{S}\left|\partial_{j}\right|^{\frac{1}{2}} u\right\|_{\mathbf{L}^{2}}+C t^{-\frac{1}{2}}\|u\|_{\mathbf{L}^{2}} \cdot
\end{aligned}
$$

Next we use the identity

$$
\begin{aligned}
\left|\partial_{j}\right|^{\frac{1}{2}}\left\langle\frac{x_{j}}{t}\right\rangle \bar{\phi}= & \left\langle\frac{x_{j}}{t}\right\rangle^{2} \bar{\phi}\left|\partial_{j}\right|^{\frac{1}{2}}\left\langle\frac{x_{j}}{t}\right\rangle^{-1}+\left[\left|\partial_{j}\right|^{\frac{1}{2}},\left\langle\frac{x_{j}}{t}\right\rangle \bar{\phi}\right] \\
& -\left\langle\frac{x_{j}}{t}\right\rangle^{2} \bar{\phi}\left[\left|\partial_{j}\right|^{\frac{1}{2}},\left\langle\frac{x_{j}}{t}\right\rangle^{-1}\right] .
\end{aligned}
$$

Applying Lemma 2.1 we find the estimates

$$
\begin{aligned}
\left\|\left[\left|\partial_{j}\right|^{\frac{1}{2}},\left\langle\frac{x_{j}}{t}\right\rangle \bar{\phi}\right] \psi\right\|_{\mathbf{L}^{2}} & \leq C\left\|\partial_{j}\left(\left\langle\frac{x_{j}}{t}\right\rangle \phi\right)\right\|_{\mathbf{L}^{\infty}}^{\frac{1}{2}}\left\|\left\langle\frac{x_{j}}{t}\right\rangle \phi\right\|_{\mathbf{L}^{\infty}}^{\frac{1}{2}}\|\psi\|_{\mathbf{L}^{2}} \\
& \leq C\left\|\left\langle\frac{x}{t}\right\rangle \phi\right\|_{\mathbf{W}_{\infty}^{1}}\|\psi\|_{\mathbf{L}^{2}}
\end{aligned}
$$


and

$$
\begin{aligned}
& \left\|\left\langle\frac{x_{j}}{t}\right\rangle^{2} \phi\left[\left|\partial_{j}\right|^{\frac{1}{2}},\left\langle\frac{x_{j}}{t}\right\rangle^{-1}\right] \psi\right\|_{\mathbf{L}^{2}} \\
& \leq C\left\|\left\langle\frac{x}{t}\right\rangle^{2} \phi\right\|\left\|_{\mathbf{L}^{\infty}}\right\| \partial_{j}\left\langle\frac{x_{j}}{t}\right\rangle^{-1}\left\|_{\mathbf{L}^{\infty}}^{\frac{1}{2}}\right\|\left\langle\frac{x_{j}}{t}\right\rangle^{-1}\left\|_{\mathbf{L}^{\infty}}^{\frac{1}{2}}\right\| \psi \|_{\mathbf{L}^{2}} \\
& \leq C t^{-\frac{1}{2}}\left\|\left\langle\frac{x}{t}\right\rangle^{2} \phi\right\|_{\mathbf{L}^{\infty}}\|\psi\|_{\mathbf{L}^{2}} .
\end{aligned}
$$

Hence

$$
\begin{aligned}
& \left\|\left|\partial_{j}\right|^{\frac{1}{2}}\left\langle\frac{x_{j}}{t}\right\rangle \overline{\phi \mathcal{S}} \mathcal{S} u\right\|_{\mathbf{L}^{2}} \\
& \quad \leq\left(\left\|\left\langle\frac{x}{t}\right\rangle^{2} \phi\right\|_{\mathbf{L}^{\infty}}+\left\|\left\langle\frac{x}{t}\right\rangle \phi\right\|_{\mathbf{W}_{\infty}^{1}}\right)\left(\left\|\left|\partial_{j}\right|^{\frac{1}{2}}\left\langle\frac{x_{j}}{t}\right\rangle^{-1} \overline{\mathcal{S}} \mathcal{S} u\right\|_{\mathbf{L}^{2}}+\|u\|_{\mathbf{L}^{2}}\right) .
\end{aligned}
$$

Substitution of (2.6) and (2.7) into (2.5) yields

$$
\begin{aligned}
& \left|\left(\mathcal{S} u, \mathcal{S} \phi \partial_{j} u\right)\right| \leq\left(\left\|\left\langle\frac{x}{t}\right\rangle^{2} \phi\right\|_{\mathbf{L}^{\infty}}+\left\|\left\langle\frac{x}{t}\right\rangle \phi\right\|_{\mathbf{W}_{\infty}^{1}}\right) \\
& \quad \times\left(\left\|\left|\partial_{j}\right|^{\frac{1}{2}}\left\langle\frac{x_{j}}{t}\right\rangle^{-1} \overline{\mathcal{S}} \mathcal{S} u\right\|_{\mathbf{L}^{2}}+\|u\|_{\mathbf{L}^{2}}\right)\left(\left\|\left\langle\frac{x_{j}}{t}\right\rangle^{-1} \mathcal{S}\left|\partial_{j}\right|^{\frac{1}{2}} u\right\|_{\mathbf{L}^{2}}+\|u\|_{\mathbf{L}^{2}}\right) .
\end{aligned}
$$

Next we represent

$$
\begin{gathered}
\left|\partial_{j}\right|^{\frac{1}{2}}\left\langle\frac{x_{j}}{t}\right\rangle^{-1} \overline{\mathcal{S}} \psi=\overline{\mathcal{S}}\left\langle\frac{x_{j}}{t}\right\rangle^{-1}\left|\partial_{j}\right|^{\frac{1}{2}} \psi+\left[\left|\partial_{j}\right|^{\frac{1}{2}}, \cosh \left(\chi_{j}\right)\left\langle\frac{x_{j}}{t}\right\rangle^{-1}\right] \psi \\
-i \sinh \left(\chi_{j}\right) \mathcal{H}_{j}\left[\left|\partial_{j}\right|^{\frac{1}{2}},\left\langle\frac{x_{j}}{t}\right\rangle^{-1}\right] \psi+i \sinh \left(\chi_{j}\right)\left[\left|\partial_{j}\right|^{\frac{1}{2}} \mathcal{H}_{j},\left\langle\frac{x_{j}}{t}\right\rangle^{-1}\right] \psi \\
-i \sinh \left(\chi_{j}\right)\left[\left|\partial_{j}\right|^{\frac{1}{2}},\left\langle\frac{x_{j}}{t}\right\rangle^{-1}\right] \mathcal{H}_{j} \psi-i\left[\left|\partial_{j}\right|^{\frac{1}{2}}, \sinh \left(\chi_{j}\right)\right]\left\langle\frac{x_{j}}{t}\right\rangle^{-1} \mathcal{H}_{j} \psi
\end{gathered}
$$

By Lemma 2.1 we get

$$
\left\|\left[\left|\partial_{j}\right|^{\frac{1}{2}}, \cosh \left(\chi_{j}\right)\left\langle\frac{x_{j}}{t}\right\rangle^{-1}\right] \psi\right\|_{\mathbf{L}^{2}}+\left\|\left[\left|\partial_{j}\right|^{\frac{1}{2}}, \sinh \left(\chi_{j}\right)\right] \psi\right\|_{\mathbf{L}^{2}} \leq C t^{-\frac{1}{2}}\|\psi\|_{\mathbf{L}^{2}}
$$

and

$$
\left\|\left[\left|\partial_{j}\right|^{\frac{1}{2}},\left\langle\frac{x_{j}}{t}\right\rangle^{-1}\right] \psi\right\|_{\mathbf{L}^{2}}+\left\|\left[\left|\partial_{j}\right|^{\frac{1}{2}} \mathcal{H}_{j},\left\langle\frac{x_{j}}{t}\right\rangle^{-1}\right] \psi\right\|_{\mathbf{L}^{2}} \leq C t^{-\frac{1}{2}}\|\psi\|_{\mathbf{L}^{2}}
$$

Therefore we have from (2.9)

$$
\left\|\left|\partial_{j}\right|^{\frac{1}{2}}\left\langle\frac{x_{j}}{t}\right\rangle^{-1} \overline{\mathcal{S}} \mathcal{S} u\right\|_{\mathbf{L}^{2}} \leq C\left\|\left\langle\frac{x_{j}}{t}\right\rangle^{-1}\left|\partial_{j}\right|^{\frac{1}{2}} \mathcal{S} u\right\|_{\mathbf{L}^{2}}+C t^{-\frac{1}{2}}\|u\|_{\mathbf{L}^{2}} .
$$

Substitution of (2.10) into (2.8) yields the result of the lemma. Lemma 2.3 is proved. 


\section{Preliminary estimates}

The following lemma was proved in paper [12]. It will be used in many stages of the prove of our result. Define the operator $\mathcal{I}=(x \cdot \nabla)-2 t \partial_{t}$, and $\Omega=x_{1} \partial_{2}-$ $x_{2} \partial_{1}$ act as the angular derivatives. Denote $\mathcal{P}=(\mathcal{I}, \Omega, t \mathcal{L}, 1)$ and $B=\left\langle\frac{x}{\sqrt{t}}\right\rangle^{-1}$.

Lemma 3.1. The estimate is true

$$
\sum_{j, k=1,2}\left\|B^{\alpha} \partial_{k} \mathcal{J}_{j} \phi\right\|_{\mathbf{L}^{2}}^{2} \leq C\left\|B^{\alpha} \mathcal{P} \phi\right\|_{\mathbf{L}^{2}}+\alpha t^{-\frac{1}{2}}\left\|B^{\alpha+1} \mathcal{J} \phi\right\|_{\mathbf{L}^{2}}
$$

for $\alpha \geq 0$.

Proof. Using the idea of the proof of Lemma 2.2 from paper [12] we obtain integrating by parts

$$
\begin{aligned}
& \sum_{j, k=1,2}\left\|B^{\alpha} \partial_{k} \mathcal{J}_{j} \phi\right\|_{\mathbf{L}^{2}}^{2}=\sum_{j, k=1,2} \int_{\mathbf{R}^{2}} \overline{\partial_{k}\left(x_{j}+i t \partial_{j}\right) \phi}\left(\partial_{k} \mathcal{J}_{j} \phi\right) B^{2 \alpha} d x \\
& =\sum_{j, k=1,2} \int_{\mathbf{R}^{2}} \overline{\left(\partial_{k} x_{j}-\partial_{j} x_{k}\right) \phi}\left(\partial_{k} \mathcal{J}_{j} \phi\right) B^{2 \alpha} d x+\sum_{j, k=1,2} \int_{\mathbf{R}^{2}} \overline{\partial_{j} \mathcal{J}_{k} \phi}\left(\partial_{k} \mathcal{J}_{j} \phi\right) B^{2 \alpha} d x \\
& =\sum_{j, k=1,2} \int_{\mathbf{R}^{2}} \overline{\left(x_{j} \partial_{k}-x_{k} \partial_{j}\right) \phi}\left(\partial_{k} \mathcal{J}_{j} \phi\right) B^{2 \alpha} d x+\sum_{j, k=1,2} \int_{\mathbf{R}^{2}} \overline{\partial_{k} \mathcal{J}_{k} \phi}\left(\partial_{j} \mathcal{J}_{j} \phi\right) B^{2 \alpha} d x \\
& \quad+\sum_{j, k=1,2} \int_{\mathbf{R}^{2}} \overline{\mathcal{J}_{k} \phi}\left(\left(\partial_{j} \mathcal{J}_{j} \phi\right) \partial_{k} B^{2 \alpha}+\left(\partial_{k} \mathcal{J}_{j} \phi\right) \partial_{j} B^{2 \alpha}\right) d x \\
& \leq C\left(\left\|B^{\alpha} \Omega \phi\right\|_{\mathbf{L}^{2}}+\alpha t^{-\frac{1}{2}}\left\|B^{\alpha+1} \mathcal{J} \phi\right\|_{\mathbf{L}^{2}}\right) \sum_{j, k=1,2}\left\|B^{\alpha} \partial_{k} \mathcal{J}_{j} \phi\right\|_{\mathbf{L}^{2}}+C\left\|B^{\alpha}(\nabla \cdot \mathcal{J}) \phi\right\|_{\mathbf{L}^{2}}^{2} .
\end{aligned}
$$

Now by the relation $(\mathcal{J} \cdot \nabla)=2 i t \mathcal{L}-\mathcal{I}$, we find the estimate

$$
\begin{aligned}
& \sum_{j, k=1,2}\left\|B^{\alpha} \partial_{k} \mathcal{J}_{j} \phi\right\|_{\mathbf{L}^{2}}^{2} \leq C\left\|B^{\alpha} \mathcal{I} \phi\right\|_{\mathbf{L}^{2}}+C\left\|B^{\alpha} \Omega \phi\right\|_{\mathbf{L}^{2}}+C t\left\|B^{\alpha} \mathcal{L} \phi\right\|_{\mathbf{L}^{2}} \\
& \quad+\left\|B^{\alpha} \phi\right\|_{\mathbf{L}^{2}}+\alpha t^{-\frac{1}{2}}\left\|B^{\alpha+1} \mathcal{J} \phi\right\|_{\mathbf{L}^{2}} \leq C\left\|B^{\alpha} \mathcal{P} \phi\right\|_{\mathbf{L}^{2}}+\alpha t^{-\frac{1}{2}}\left\|B^{\alpha+1} \mathcal{J} \phi\right\|_{\mathbf{L}^{2}} .
\end{aligned}
$$

Lemma 3.1 is proved.

In the next lemma we estimate different norms by the norm

$$
\|u\|_{\mathbf{X}_{T}}=\sup _{t \in[1, T]}\left(t^{-\gamma}\left\|\Gamma^{3} u\right\|_{\mathbf{L}^{2}}+t^{-3 \gamma}\left\|\mathcal{J} \Gamma^{2} u\right\|_{\mathbf{L}^{2}}+\left\|\Gamma^{2} u\right\|_{\mathbf{L}^{2}}\right)
$$

where $\gamma \in\left(0, \frac{1}{6}\right), \Gamma=(1, \mathcal{I}, \Omega, \Delta), \mathcal{I}=(x \cdot \nabla)-2 t \partial_{t}$ and $\Omega=x_{2} \partial_{1}-x_{1} \partial_{2}$.

Lemma 3.2. Assume that the norm $\|u\|_{\mathbf{X}_{T}}$ is finite. Then for the solutions of Eq. (1.1) the estimates are valid

$$
\begin{aligned}
& t^{1-\frac{2}{p}}\|\nabla \Gamma u\|_{\mathbf{L}^{p}}+t^{(1-\gamma)\left(1-\frac{2}{p}\right)}\|\nabla \Gamma u\|_{\mathbf{W}_{p}^{1}}+t^{1-\frac{2}{p}-\gamma}\left\|\Gamma^{2} u\right\|_{\mathbf{W}_{p}^{1}} \\
& \quad+\|\mathcal{J} \nabla \Gamma u\|_{\mathbf{L}^{2}}+t^{-\gamma}\left\|\mathcal{J} \nabla \Gamma^{2} u\right\|_{\mathbf{L}^{2}} \leq C \varepsilon
\end{aligned}
$$

and 


$$
\begin{aligned}
& t^{\frac{3}{2}-\frac{2}{p}}\|B \Delta u\|_{\mathbf{L}^{p}}+t^{\frac{3}{2}-\frac{2}{p}-\gamma}\left\|B \Delta \Gamma^{2} u\right\|_{\mathbf{L}^{p}}+t^{1-\frac{1}{p}-\gamma}\left\|B \nabla \Gamma^{2} u\right\|_{\mathbf{L}^{p}} \\
& \quad+t^{\frac{1}{2}}\|B \mathcal{J} \Delta \Gamma u\|_{\mathbf{L}^{2}}+t^{\frac{1}{2}-\frac{1}{p}}\|B \mathcal{J} \nabla \Gamma u\|_{\mathbf{L}^{p}}+t^{-\frac{1}{2}}\left\|B \mathcal{J}^{2} \nabla \Gamma u\right\|_{\mathbf{L}^{2}} \leq C \varepsilon
\end{aligned}
$$

for $t \in[1, T]$, where $2 \leq p<\infty$, and $\varepsilon=\|u\|_{\mathbf{X}_{T}}\left(1+\|u\|_{\mathbf{X}_{T}}^{3}\right)$.

Proof. By the Sobolev imbedding theorem we have $\|\phi\|_{\mathbf{L}^{p}} \leq C\|\phi\|_{\mathbf{L}^{q}}^{1-\alpha}\left\|\partial^{m} \phi\right\|_{\mathbf{L}^{r}}^{\alpha}$ for $1 \leq p, q, r \leq \infty, m \in \mathbf{N}$ and $\alpha \in[0,1)$ such that $\frac{1}{p}=\alpha\left(\frac{1}{r}-\frac{1}{q}-\frac{m}{n}\right)+\frac{1}{q}$. Therefore we get

$$
\begin{aligned}
\|\phi\|_{\mathbf{L}^{p}} & =\|\bar{M} \phi\|_{\mathbf{L}^{p}} \leq C\|\bar{M} \phi\|_{\mathbf{L}^{2}}^{\frac{2}{p}}\|\nabla \bar{M} \phi\|_{\mathbf{L}^{2}}^{1-\frac{2}{p}} \\
& =C t^{\frac{2}{p}-1}\|\phi\|_{\mathbf{L}^{2}}^{\frac{2}{p}}\|\mathcal{J} \phi\|_{\mathbf{L}^{2}}^{1-\frac{2}{p}} \leq C t^{\frac{2}{p}-1}\|\phi\|_{\mathbf{L}^{2}}^{\frac{2}{p}}\|\mathcal{J} \phi\|_{\mathbf{L}^{2}}^{1-\frac{2}{p}}
\end{aligned}
$$

for $2 \leq p<\infty$. Then

$$
t\|\mathcal{L} \Gamma u\|_{\mathbf{L}^{2}} \leq t\|\widetilde{\Gamma} \mathcal{N}(\nabla u, \nabla u)\|_{\mathbf{L}^{2}} \leq C t\|\nabla u\|_{\mathbf{L}^{\infty}}\|\Gamma \nabla u\|_{\mathbf{L}^{2}}
$$

We can use a rough estimate

$$
\begin{aligned}
\|\nabla u\|_{\mathbf{W}_{\infty}^{1}} & \leq C\|\nabla u\|_{\mathbf{W}_{p}^{2}} \leq C t^{\frac{2}{p}-1}\|\nabla u\|_{\mathbf{H}^{2}}^{\frac{2}{p}}\|\mathcal{J} \nabla u\|_{\mathbf{H}^{2}}^{1-\frac{2}{p}} \leq C t^{\frac{2}{p}-1}\left\|\Gamma^{2} u\right\|_{\mathbf{L}^{2}} \\
& \leq C \varepsilon t^{\gamma-1}
\end{aligned}
$$

by Lemma 3.1 we get

$$
\begin{aligned}
t^{1-\frac{2}{p}}\|\nabla \Gamma u\|_{\mathbf{L}^{p}} & \leq C\|\nabla \Gamma u\|_{\mathbf{L}^{2}}^{\frac{1}{2}}\|\mathcal{J} \nabla \Gamma u\|_{\mathbf{L}^{2}}^{\frac{1}{2}} \\
& \leq C\|\nabla \Gamma u\|_{\mathbf{L}^{2}}^{\frac{1}{2}}\left(\left\|\Gamma^{2} u\right\|_{\mathbf{L}^{2}}+t\|\mathcal{L} \Gamma u\|_{\mathbf{L}^{2}}\right)^{\frac{1}{2}} \leq C \varepsilon .
\end{aligned}
$$

In the same manner

$$
\begin{aligned}
\left\|\Gamma^{2} u\right\|_{\mathbf{L}^{p}} \leq & C t^{\frac{2}{p}-1}\left\|\Gamma^{2} u\right\|_{\mathbf{L}^{2}}^{\frac{2}{p}}\left\|\mathcal{J} \Gamma^{2} u\right\|_{\mathbf{L}^{2}}^{1-\frac{2}{p}} \leq C \varepsilon^{2} t^{\gamma+\frac{2}{p}-1}, \\
& \times t\left\|\mathcal{L} \Gamma^{2} u\right\|_{\mathbf{L}^{2}} \leq t\left\|\widetilde{\Gamma}^{2} \mathcal{N}(\nabla u, \nabla u)\right\|_{\mathbf{L}^{2}} \\
\leq & C t\|\nabla \Gamma u\|_{\mathbf{L}^{4}}^{2}+C t\|\nabla u\|_{\mathbf{W}_{\infty}^{1}}\left\|\nabla \Gamma^{2} u\right\|_{\mathbf{L}^{2}} \leq C \varepsilon t^{\gamma}
\end{aligned}
$$

and by Lemma 3.1 we obtain

$$
\begin{aligned}
& t^{1-\frac{2}{p}-\gamma}\left\|\nabla \Gamma^{2} u\right\|_{\mathbf{L}^{p}} \leq C t^{-\gamma}\left\|\nabla \Gamma^{2} u\right\|_{\mathbf{L}^{2}}^{\frac{2}{p}}\left\|\mathcal{J} \nabla \Gamma^{2} u\right\|_{\mathbf{L}^{2}}^{1-\frac{2}{p}} \\
& \quad \leq C t^{-\gamma}\left\|\nabla \Gamma^{2} u\right\|_{\mathbf{L}^{2}}^{\frac{2}{p}}\left(\left\|\Gamma^{3} u\right\|_{\mathbf{L}^{2}}+t\left\|\mathcal{L} \Gamma^{2} u\right\|_{\mathbf{L}^{2}}\right)^{1-\frac{2}{p}} \\
& \quad \leq C t^{-\gamma}\left\|\Gamma^{3} u\right\|_{\mathbf{L}^{2}}+C \varepsilon^{2} \leq C \varepsilon .
\end{aligned}
$$

In the same manner we get

$$
\begin{aligned}
& t^{(1-\gamma)\left(1-\frac{2}{p}\right)}\|\Delta \Gamma u\|_{\mathbf{L}^{p}} \leq C t^{-\gamma\left(1-\frac{2}{p}\right)}\|\Delta \Gamma u\|_{\mathbf{L}^{2}}^{\frac{2}{p}}\|\mathcal{J} \Delta \Gamma u\|_{\mathbf{L}^{2}}^{1-\frac{2}{p}} \\
& \quad \leq C t^{-\gamma\left(1-\frac{2}{p}\right)}\left\|\Gamma^{2} u\right\|_{\mathbf{L}^{2}}^{\frac{2}{p}}\left(\left\|\nabla \Gamma^{2} u\right\|_{\mathbf{L}^{2}}+t\|\mathcal{L} \nabla \Gamma u\|_{\mathbf{L}^{2}}\right)^{1-\frac{2}{p}} \leq C \varepsilon .
\end{aligned}
$$

Also by Lemma Lemma 3.1 we find

$$
\|\mathcal{J} \nabla \Gamma u\|_{\mathbf{L}^{2}} \leq\left\|\Gamma^{2} u\right\|_{\mathbf{L}^{2}}+C t\|\mathcal{L} \Gamma u\|_{\mathbf{L}^{2}} \leq C \varepsilon^{2}
$$

and 


$$
\left\|\mathcal{J} \nabla \Gamma^{2} u\right\|_{\mathbf{L}^{2}} \leq\left\|\Gamma^{3} u\right\|_{\mathbf{L}^{2}}+C t\left\|\mathcal{L} \Gamma^{2} u\right\|_{\mathbf{L}^{2}} \leq C t^{\gamma} \varepsilon^{2} .
$$

Thus the first estimate of the lemma is true.

Next we consider estimates involving $B$. By the identity $\nabla=i t^{-1}(x-\mathcal{J})$ and the first estimate of the lemma we obtain

$$
\begin{gathered}
\left\|B \nabla \Gamma^{2} u\right\|_{\mathbf{L}^{2}} \leq C t^{-1}\left\|B x \Gamma^{2} u\right\|_{\mathbf{L}^{2}}+C t^{-1}\left\|\mathcal{J} \Gamma^{2} u\right\|_{\mathbf{L}^{2}} \leq C \varepsilon t^{-\frac{1}{2}} \\
\left\|B \Delta \Gamma^{2} u\right\|_{\mathbf{L}^{2}} \leq C t^{-1}\left\|B x \nabla \Gamma^{2} u\right\|_{\mathbf{L}^{2}}+C t^{-1}\left\|B \mathcal{J} \nabla \Gamma^{2} u\right\|_{\mathbf{L}^{2}} \leq C \varepsilon t^{\gamma-\frac{1}{2}} \\
\|B \mathcal{J} \Delta u\|_{\mathbf{L}^{2}} \leq C\|B \nabla \Gamma u\|_{\mathbf{L}^{2}}+C t\|B \mathcal{L} \nabla \Gamma u\|_{\mathbf{L}^{2}}+t^{-\frac{1}{2}}\left\|B^{2} \mathcal{J} \nabla u\right\|_{\mathbf{L}^{2}} \leq C \varepsilon t^{-\frac{1}{2}}
\end{gathered}
$$

Hence by the Sobolev imbedding Theorem (3.1) and the first estimate of the lemma we get

$$
\begin{gathered}
\|B \Delta u\|_{\mathbf{L}^{p}} \leq C t^{\frac{2}{p}-1}\|B \Delta u\|_{\mathbf{L}^{2}}^{\frac{2}{p}}\|B \mathcal{J} \Delta u\|_{\mathbf{L}^{2}}^{1-\frac{2}{p}} \leq C \varepsilon t^{\frac{2}{p}-\frac{3}{2}} \\
\left\|B \Delta \Gamma^{2} u\right\|_{\mathbf{L}^{p}} \leq C t^{\frac{2}{p}-1}\left\|B \Delta \Gamma^{2} u\right\|_{\mathbf{L}^{2}}^{\frac{2}{p}}\left\|B \mathcal{J} \Delta \Gamma^{2} u\right\|_{\mathbf{L}^{2}}^{1-\frac{2}{p}} \leq C \varepsilon t^{\frac{2}{p}-\frac{3}{2}+\gamma}, \\
\|B \nabla \Gamma u\|_{\mathbf{L}^{p}} \leq C t^{\frac{2}{p}-1}\|B \nabla \Gamma u\|_{\mathbf{L}^{2}}^{\frac{2}{p}}\|\mathcal{J} \nabla \Gamma u\|_{\mathbf{L}^{2}}^{1-\frac{2}{p}} \leq C \varepsilon t^{\frac{1}{p}-1}
\end{gathered}
$$

and

$$
\left\|B \nabla \Gamma^{2} u\right\|_{\mathbf{L}^{p}} \leq C t^{\frac{2}{p}-1}\left\|B \nabla \Gamma^{2} u\right\|_{\mathbf{L}^{2}}^{\frac{2}{p}}\left\|\mathcal{J} \nabla \Gamma^{2} u\right\|_{\mathbf{L}^{2}}^{1-\frac{2}{p}} \leq C \varepsilon t^{\frac{1}{p}-1+\gamma\left(1-\frac{2}{p}\right)} .
$$

Then by Lemma 3.1 we obtain

$$
\begin{gathered}
\|B \mathcal{J} \Delta \Gamma u\|_{\mathbf{L}^{2}} \leq\left\|B \nabla \Gamma^{2} u\right\|_{\mathbf{L}^{2}}+C t\|B \nabla \mathcal{L} \Gamma u\|_{\mathbf{L}^{2}}+t^{-\frac{1}{2}}\left\|B^{2} \mathcal{J} \nabla \Gamma u\right\|_{\mathbf{L}^{2}} \leq C \varepsilon t^{-\frac{1}{2}}, \\
\|B \mathcal{J} \nabla \Gamma u\|_{\mathbf{L}^{p}} \leq C t^{\frac{2}{p}-1}\|\mathcal{J} \nabla \Gamma u\|_{\mathbf{L}^{2}}^{\frac{2}{p}}\left\|B \mathcal{J}^{2} \nabla \Gamma u\right\|_{\mathbf{L}^{2}}^{1-\frac{2}{p}} \leq C \varepsilon t^{\frac{1}{p}-\frac{1}{2}}
\end{gathered}
$$

and

$$
\begin{aligned}
& \left\|B \mathcal{J}^{2} \nabla \Gamma u\right\|_{\mathbf{L}^{2}} \leq C\left\|B \mathcal{J} \Gamma^{2} u\right\|_{\mathbf{L}^{2}}+C t\|B \mathcal{J} \mathcal{L} \Gamma u\|_{\mathbf{L}^{2}} \\
& \leq C t^{\gamma}\|u\|_{\mathbf{X}_{T}}+C t\|\nabla u\|_{\mathbf{L}^{\infty}}\|B \mathcal{J} \nabla \Gamma u\|_{\mathbf{L}^{2}} \\
& \quad+C t\|B \nabla \Gamma u\|_{\mathbf{L}^{\infty}}\|\mathcal{J} \nabla u\|_{\mathbf{L}^{2}}+C t\|\nabla u\|_{\mathbf{L}^{\infty}}\|B x \nabla \Gamma u\|_{\mathbf{L}^{2}} \leq C \varepsilon t^{\frac{1}{2}} .
\end{aligned}
$$

Lemma 3.2 is proved.

In the next lemma we establish estimates for the norms $\left\|\left\langle\frac{x}{t}\right\rangle^{2} \nabla u\right\|_{\mathbf{L}^{\infty}}$ and $\left\|\left\langle\frac{x}{t}\right\rangle \nabla u\right\|_{\mathbf{W}_{\infty}^{1}}$.

Lemma 3.3. Let the initial data $u_{0} \in \mathbf{H}^{6} \cap \mathbf{H}^{0,3}$. Assume that for the solutions of Eq. (1.1) the estimate $\|u\|_{\mathbf{X}_{T}} \leq C \varepsilon$ is true. Then the estimate is valid

$$
\left\|\left\langle\frac{x}{t}\right\rangle^{2} \nabla u\right\|_{\mathbf{L}^{\infty}}+\left\|\left\langle\frac{x}{t}\right\rangle \nabla u\right\|_{\mathbf{W}_{\infty}^{1}} \leq C \varepsilon
$$

for $t \in[1, T]$. 
Proof. First we estimate the norm $\|\nabla u\|_{\mathbf{w}_{\infty}^{1}}$. We change $u=h-t B^{2} \mathcal{N}$ $(\nabla u, \nabla u)$, then by the relation $\nabla=i t^{-1}(x-\mathcal{J})$ we get

$$
\|\nabla u\|_{\mathbf{W}_{\infty}^{1}} \leq\|\nabla h\|_{\mathbf{W}_{\infty}^{1}}+\left\|\nabla t B^{2} \mathcal{N}(\nabla u, \nabla u)\right\|_{\mathbf{W}_{\infty}^{1}} .
$$

By the relation $\nabla=i t^{-1}(x-\mathcal{J})$ we find

$$
\begin{aligned}
& \left\|\nabla t B^{2} \mathcal{N}(\nabla u, \nabla u)\right\|_{\mathbf{W}_{\infty}^{1}} \\
& \quad \leq C\left\|x B^{2} \mathcal{N}(\nabla u, \nabla u)\right\|_{\mathbf{W}_{\infty}^{1}}+C t^{-1}\left\|t B^{2} \mathcal{J N}(\nabla u, \nabla u)\right\|_{\mathbf{W}_{\infty}^{1}} \\
& \quad \leq C t^{\frac{1}{2}}\|\nabla u\|_{\mathbf{W}_{\infty}^{1}}^{2}+C\|\nabla u\|_{\mathbf{W}_{\infty}^{1}}\left\|B^{2} \mathcal{J} \nabla u\right\|_{\mathbf{W}_{\infty}^{1}} .
\end{aligned}
$$

We can use a rough estimate $\|\nabla u\|_{\mathbf{W}_{\infty}^{1}} \leq C \varepsilon t^{\frac{2}{p}-1}$, then by the Sobolev inequality and Lemma 3.1 we obtain

$$
\left\|B^{2} \mathcal{J} \nabla u\right\|_{\mathbf{W}_{\infty}^{1}} \leq C t^{-\frac{1}{4}}\left\|B^{2} \mathcal{J} \nabla u\right\|_{\mathbf{H}^{2}}^{\frac{3}{2}}\left\|B^{2} \mathcal{J}^{2} \Delta u\right\|_{\mathbf{H}^{1}}^{\frac{1}{4}}
$$

By the relation $\nabla=i t^{-1}(x-\mathcal{J})$ we get

$$
\begin{aligned}
\left\|B^{2} \Delta \mathcal{J}^{2} u\right\|_{\mathbf{H}^{1}} \leq C\left\|B^{2} \mathcal{J} \nabla \Gamma u\right\|_{\mathbf{H}^{1}}+C t\left\|B^{2} \mathcal{L} \mathcal{J} \nabla u\right\|_{\mathbf{H}^{1}} \\
\quad+C t^{-\frac{1}{2}}\left\|B^{2}(x+i t \nabla) \mathcal{J} \nabla u\right\|_{\mathbf{H}^{1}} \\
\leq C\left\|\Gamma^{2} u\right\|_{\mathbf{H}^{1}}+C t\left\|B^{2} \mathcal{L} \Gamma u\right\|_{\mathbf{H}^{1}}+C t\|\nabla u\|_{\mathbf{W}_{\infty}^{1}}\|\mathcal{J} \Delta u\|_{\mathbf{H}^{1}} \\
\quad+C t\|\nabla u\|_{\mathbf{W}_{\infty}^{1}}\left\|x B^{2} \Delta u\right\|_{\mathbf{H}^{1}} \\
\leq C \varepsilon t^{\gamma}+C \varepsilon t^{-1}\left\||x|^{2} B^{2} \nabla u\right\|_{\mathbf{H}^{1}} \leq C \varepsilon t^{\gamma} .
\end{aligned}
$$

Hence $\left\|\nabla t B^{2} \mathcal{N}(\nabla u, \nabla u)\right\|_{\mathbf{W}_{\infty}^{1}} \leq C \varepsilon^{2} t^{-1}$. So we need to obtain an optimal time decay estimate for $\|\nabla h\|_{\mathbf{w}_{\infty}^{1}}$. By the Sobolev inequality we obtain

$$
\begin{aligned}
\|\nabla h\|_{\mathbf{W}_{\infty}^{1}} & =\|\bar{M} \nabla h\|_{\mathbf{W}_{\infty}^{1}} \leq C\|\bar{M} \nabla h\|_{\mathbf{H}^{1}}^{\frac{1}{2}}\|\Delta \bar{M} \nabla h\|_{\mathbf{H}^{1}}^{\frac{1}{2}} \\
& =C t^{-1}\|\nabla h\|_{\mathbf{H}^{1}}^{\frac{1}{2}}\left\|\mathcal{J}^{2} \nabla h\right\|_{\mathbf{H}^{1}}^{\frac{1}{2}} .
\end{aligned}
$$

By Lemma 3.1 we find the estimate

$$
\left\|\mathcal{J}^{2} \nabla h\right\|_{\mathbf{H}^{1}} \leq C\|\Gamma \mathcal{J} h\|_{\mathbf{H}^{1}}+C t\|\mathcal{L} \mathcal{J} h\|_{\mathbf{H}^{1}} \text {. }
$$

Since $\|\Gamma \mathcal{J} \nabla h\|_{\mathbf{L}^{2}} \leq\|\Gamma \mathcal{J} \nabla u\|_{\mathbf{L}^{2}}+\left\|\Gamma \mathcal{J} \nabla t B^{2} \mathcal{N}(\nabla u, \nabla u)\right\|_{\mathbf{L}^{2}} \leq\left\|\Gamma^{2} u\right\|_{\mathbf{L}^{2}}+$ $C t\|\mathcal{L} \Gamma u\|_{\mathbf{L}^{2}}$, so we need to estimate the norms $\|\mathcal{J} \Gamma h\|_{\mathbf{L}^{2}}$ and $\|\mathcal{L} \mathcal{J} h\|_{\mathbf{H}^{1}}$. By Lemma 3.5 we have $\left\|\mathcal{L} \Gamma^{2} h\right\|_{\mathbf{L}^{2}} \leq C \varepsilon^{2} t^{2 \gamma-\frac{4}{3}}$.

Denote $\mathcal{J}^{m}=M(i t \nabla)^{m} \bar{M}$. By the Sobolev inequality $\|\phi\|_{\mathbf{L}^{p}} \leq$ $C\|\phi\|_{\mathbf{L}^{q}}^{1-\alpha}\left\|\partial^{m} \phi\right\|_{\mathbf{L}^{r}}^{\alpha}$ for $1 \leq p, q, r \leq \infty, m \in \mathbf{N}$ and $\alpha \in[0,1)$ such that $\frac{1}{p}=\alpha\left(\frac{1}{r}-\frac{1}{q}-\frac{m}{n}\right)+\frac{1}{q}$ we get

$$
\begin{aligned}
\|\nabla h\|_{\mathbf{L}^{\infty}} & =\|\bar{M} \nabla h\|_{\mathbf{L}^{\infty}} \leq C\|\bar{M} \nabla h\|_{\mathbf{L}^{2}}^{\frac{1}{3}}\left\|(i t \nabla)^{\frac{3}{2}} \bar{M} \nabla h\right\|_{\mathbf{L}^{2}}^{\frac{2}{3}} \\
& =C t^{-1}\|\nabla h\|_{\mathbf{L}^{2}}^{\frac{1}{3}}\left\|\mathcal{J}^{\frac{3}{2}} \nabla h\right\|_{\mathbf{L}^{2}}^{\frac{2}{3}} .
\end{aligned}
$$


We can estimate $\left\|\mathcal{J}^{\frac{3}{2}} \nabla h\right\|_{\mathbf{L}^{2}} \leq\left\|\mathcal{J}^{\frac{1}{2}} \Gamma h\right\|_{\mathbf{L}^{2}}+t\left\|\mathcal{J}^{\frac{1}{2}} \mathcal{L} h\right\|_{\mathbf{L}^{2}}$ and

$$
\begin{aligned}
\frac{d}{d t}\left\|\mathcal{J}^{\frac{1}{2}} \Gamma h\right\|_{\mathbf{L}^{2}}^{2} & =2 \operatorname{Im}\left(\mathcal{J}^{\frac{1}{2}} \Gamma h, \mathcal{J}^{\frac{1}{2}} \mathcal{L} \Gamma h\right) \\
& =2 \operatorname{Im}(\mathcal{J} \Gamma h, \mathcal{L} \Gamma h) \leq 2\|\mathcal{J} \Gamma h\|_{\mathbf{L}^{2}}\|\mathcal{L} \Gamma h\|_{\mathbf{L}^{2}} \leq C \varepsilon^{2} t^{3 \gamma-\frac{4}{3}}
\end{aligned}
$$

Hence integrating we obtain $\left\|\mathcal{J}^{\frac{1}{2}} \Gamma h\right\|_{\mathbf{L}^{2}}^{2} \leq C \varepsilon^{2}$. In the same manner by Lemma 3.5

$$
\begin{aligned}
\left\|\mathcal{J}^{\frac{1}{2}} \mathcal{L} h\right\|_{\mathbf{L}^{2}}^{2} & =\left(\mathcal{J}^{\frac{1}{2}} \mathcal{L} h, \mathcal{J}^{\frac{1}{2}} \mathcal{L} h\right)=(\mathcal{J} \mathcal{L} h, \mathcal{L} h) \\
& \leq 2\|\mathcal{J} \mathcal{L} h\|_{\mathbf{L}^{2}}\|\mathcal{L} h\|_{\mathbf{L}^{2}} \leq C \varepsilon^{4} t^{7 \gamma-\frac{7}{3}}
\end{aligned}
$$

Hence we find

$$
\left\|\mathcal{J}^{\frac{3}{2}} \nabla h\right\|_{\mathbf{L}^{2}} \leq\left\|\mathcal{J}^{\frac{1}{2}} \Gamma h\right\|_{\mathbf{L}^{2}}+t\left\|\mathcal{J}^{\frac{1}{2}} \mathcal{L} h\right\|_{\mathbf{L}^{2}} \leq C \varepsilon+C \varepsilon^{2} t^{4 \gamma-\frac{1}{6}} \leq C \varepsilon .
$$

Therefore we get an optimal time decay estimate

$$
\|\nabla h\|_{\mathbf{L}^{\infty}} \leq C \varepsilon t^{-1} .
$$

We have

$$
\begin{aligned}
& \left\|\left\langle\frac{x}{t}\right\rangle^{2} \nabla u\right\|_{\mathbf{L}^{\infty}}+\left\|\left\langle\frac{x}{t}\right\rangle \nabla u\right\|_{\mathbf{W}_{\infty}^{1}} \\
& \quad \leq C\|\nabla u\|_{\mathbf{W}_{\infty}^{1}}+C t^{-1}\|x \Delta u\|_{\mathbf{L}^{\infty}}+C t^{-2}\left\||x|^{2} \nabla u\right\|_{\mathbf{L}^{\infty}} .
\end{aligned}
$$

For the last summand by the relation $t^{-1} x=-i \nabla+t^{-1} \mathcal{J}$ we find

$$
t^{-2}\left\||x|^{2} \nabla u\right\|_{\mathbf{L}^{\infty}} \leq C t^{-2}\|x \mathcal{J} \nabla u\|_{\mathbf{L}^{\infty}}+C t^{-1}\|x \Delta u\|_{\mathbf{L}^{\infty}} .
$$

Then changing $u=h-t B^{2} \mathcal{N}(\nabla u, \nabla u)$, by the relation $t^{-1} x=-i \nabla+t^{-1} \mathcal{J}$ we obtain

$$
\begin{aligned}
& \left\|\frac{x}{t} \nabla \mathcal{J} u\right\|_{\mathbf{L}^{\infty}} \leq\left\|\frac{x}{t} \nabla \mathcal{J} h\right\|_{\mathbf{L}^{\infty}}+\left\|x \nabla \mathcal{J} B^{2} \mathcal{N}(\nabla u, \nabla u)\right\|_{\mathbf{L}^{\infty}} \\
& \quad \leq\|\Delta \mathcal{J} h\|_{\mathbf{L}^{\infty}}+t^{-1}\left\|\nabla \mathcal{J}^{2} h\right\|_{\mathbf{L}^{\infty}}+C t^{\frac{1}{2}}\|\nabla u\|_{\mathbf{W}_{\infty}^{1}}\|B \mathcal{J} \nabla u\|_{\mathbf{W}_{\infty}^{1}} \\
& \quad \leq C t^{-\frac{1}{2}}\|\Delta \mathcal{J} h\|_{\mathbf{H}^{1}}^{\frac{1}{2}}\left\|\Delta \mathcal{J}^{2} h\right\|_{\mathbf{H}^{1}}^{\frac{1}{2}}+t^{-1}\left\|\nabla \mathcal{J}^{2} h\right\|_{\mathbf{H}^{2}}+C \varepsilon^{2} \\
& \quad \leq C t^{-\frac{1}{2}}\|\Delta \mathcal{J} h\|_{\mathbf{H}^{1}}^{\frac{1}{2}}\left\|\Delta \mathcal{J}^{2} h\right\|_{\mathbf{H}^{1}}^{\frac{1}{2}}+t^{-1}\|\mathcal{J} \Gamma h\|_{\mathbf{H}^{2}}+\|\mathcal{J} \mathcal{L} h\|_{\mathbf{H}^{2}}+C \varepsilon^{2} \\
& \quad \leq C t^{-\frac{1}{2}}\left\|\mathcal{J} \Gamma^{2} h\right\|_{\mathbf{L}^{2}}+C \varepsilon^{2} .
\end{aligned}
$$

By Lemma 3.2 we have $\|\nabla \Gamma u\|_{\mathbf{L}^{\infty}} \leq C\|\nabla \Gamma u\|_{\mathbf{W}_{2 / \gamma}^{1}} \leq C \varepsilon t^{2 \gamma-1}$ and $\left\|B \nabla \Gamma^{2} u\right\|_{\mathbf{L}^{2}} \leq C \varepsilon t^{-\frac{1}{2}}$. Then since $\|\nabla u\|_{\mathbf{W}_{\infty}^{1}} \leq C \varepsilon t^{\frac{2}{p}-1}$ we get

$$
\left\|\Gamma^{2} x t B^{2} \mathcal{N}(\nabla u, \nabla u)\right\|_{\mathbf{L}^{2}} \leq C t^{\frac{3}{2}}\|\nabla \Gamma u\|_{\mathbf{L}^{\infty}}\left\|B \nabla \Gamma^{2} u\right\|_{\mathbf{L}^{2}} \leq C \varepsilon^{2} t^{2 \gamma} .
$$

Hence

$$
\left\|\mathcal{J} \Gamma^{2} h\right\|_{\mathbf{L}^{2}} \leq\left\|\mathcal{J} \Gamma^{2} u\right\|_{\mathbf{L}^{2}}+\left\|\mathcal{J} \Gamma^{2} t B^{2} \mathcal{N}(\nabla u, \nabla u)\right\|_{\mathbf{L}^{2}} \leq C \varepsilon^{2} t^{3 \gamma} .
$$


Thus we need to estimate the norm $t^{-1}\|x \Delta u\|_{\mathbf{L}^{\infty}}$. We change $u=h-$ $t B^{2} \mathcal{N}(\nabla u, \nabla u)$, then

$$
\left\|\frac{x}{t} \Delta u\right\|_{\mathbf{L}^{\infty}} \leq\left\|\frac{x}{t} \Delta h\right\|_{\mathbf{L}^{\infty}}+C\left\|x \Delta B^{2} \mathcal{N}(\nabla u, \nabla u)\right\|_{\mathbf{L}^{\infty}} .
$$

We have $\left\|x \Delta B^{2} \mathcal{N}(\nabla u, \nabla u)\right\|_{\mathbf{L}^{\infty}} \leq C \sqrt{t}\|\nabla u\|_{\mathbf{W}_{\infty}^{1}}\|\nabla \Gamma u\|_{\mathbf{L}^{\infty}} \leq C \varepsilon^{2} t^{-1}$. By the Sobolev imbedding inequality (3.1)

$$
\left\|\frac{x}{t} \Delta h\right\|_{\mathbf{L}^{\infty}} \leq C t^{-\frac{5}{6}}\left\|\frac{x}{t} \Delta h\right\|_{\mathbf{L}^{2}}^{\frac{1}{6}}\left\|\mathcal{J} \frac{x}{t} \Delta h\right\|_{\mathbf{L}^{2}}^{\frac{1}{3}}\left\|\mathcal{J} \frac{x}{t} \Delta h\right\|_{\mathbf{L}^{3}}^{\frac{1}{2}} .
$$

Then by the relation $t^{-1} x=-i \nabla+t^{-1} \mathcal{J}$ we find

$$
\begin{gathered}
\left\|\frac{x}{t} \Delta h\right\|_{\mathbf{L}^{2}} \leq\|\nabla h\|_{\mathbf{H}^{2}}+C t^{-1}\|\mathcal{J} \nabla h\|_{\mathbf{H}^{1}} \leq C \varepsilon \\
\left\|\mathcal{J} \frac{x}{t} \Delta h\right\|_{\mathbf{L}^{2}} \leq\|\mathcal{J} \nabla h\|_{\mathbf{H}^{2}}+C t^{-1}\left\|\mathcal{J}^{2} \nabla h\right\|_{\mathbf{H}^{1}} \leq C \varepsilon+C t^{-1}\left\|\mathcal{J}^{2} \nabla h\right\|_{\mathbf{H}^{1}}
\end{gathered}
$$

and

$$
\begin{aligned}
\left\|\mathcal{J} \frac{x}{t} \Delta h\right\|_{\mathbf{L}^{3}} & \leq C\|\mathcal{J} \Delta h\|_{\mathbf{W}_{3}^{1}}+C t^{-1}\left\|\mathcal{J}^{2} \Delta h\right\|_{\mathbf{L}^{3}} \\
& \leq C t^{-\frac{1}{3}}\|\mathcal{J} \Delta h\|_{\mathbf{H}^{1}}^{\frac{1}{3}}\left\|\mathcal{J}^{\frac{3}{2}} \Delta h\right\|_{\mathbf{H}^{1}}^{\frac{2}{3}}+C t^{-1}\left\|\mathcal{J}^{2} \Delta h\right\|_{\mathbf{H}^{1}}
\end{aligned}
$$

since $\|\mathcal{J} \Delta h\|_{\mathbf{H}^{1}} \leq C \varepsilon$ and $\left\|\mathcal{J}^{\frac{3}{2}} \Delta h\right\|_{\mathbf{H}^{1}} \leq C \varepsilon$. We only need a rough estimate for

$$
\left\|\mathcal{J}^{2} \nabla h\right\|_{\mathbf{H}^{2}} \leq\|\mathcal{J} \Gamma h\|_{\mathbf{H}^{2}}+C t\|\mathcal{J} \mathcal{L} h\|_{\mathbf{H}^{2}} \leq C \varepsilon t^{\gamma}
$$

since by Lemma 3.5 we have $\|\mathcal{L} \mathcal{J} \Gamma h\|_{\mathbf{L}^{2}} \leq C \varepsilon^{2} t^{\gamma-1}$. Hence we obtain

$$
\left\|\mathcal{J} \frac{x}{t} \Delta h\right\|_{\mathbf{L}^{3}} \leq C \varepsilon t^{-\frac{1}{3}}
$$

Thus

$$
\left\|\frac{x}{t} \Delta h\right\|_{\mathbf{L}^{\infty}} \leq C \varepsilon t^{-1}
$$

Therefore the result of the lemma follows. Lemma 3.3 is proved.

In the next lemma we estimate the norm $\left\|\Gamma^{3} u\right\|_{\mathbf{L}^{2}}$.

Lemma 3.4. Let the initial data $u_{0} \in \mathbf{H}^{6} \cap \mathbf{H}^{0,3}$. Assume that for the solutions of Eq. (1.1) the estimate $\|u\|_{\mathbf{X}_{T}} \leq C \varepsilon$ is true. Then the estimate is valid

$$
\left\|\Gamma^{3} u\right\|_{\mathbf{L}^{2}} \leq C t^{\gamma}\left\|u_{0}\right\|_{\mathbf{H}^{6} \cap \mathbf{H}^{0,3}}
$$

for $t \in[1, T]$, where $\gamma=C \varepsilon>0$ is small.

Proof. Applying the operator $\Gamma^{3}$ to Eq. (1.1) and using the commutator relation $[\mathcal{L}, \mathcal{I}]=2$, we get

$$
\mathcal{L} v=\widetilde{\Gamma}^{3} \mathcal{N}(\nabla u, \nabla u),
$$

where $\widetilde{\Gamma}=(1, \mathcal{I}+2, \Omega, \Delta)$ and $v=\Gamma^{3} u$. Note that the nonlinearity of equation (3.2) can be represented as follows

$$
\widetilde{\Gamma}^{3} \mathcal{N}(\nabla u, \nabla u)=2 \mathcal{N}(\nabla u, \nabla v)+R
$$


where the term $2 \mathcal{N}(\nabla u, \nabla v)$ contains a derivative loss and the remainder term

$$
\begin{aligned}
R & =\widetilde{\Gamma}^{3} \mathcal{N}(\nabla u, \nabla u)-2 \mathcal{N}(\nabla u, \nabla v) \\
& =\sum_{k, l=1,2} \lambda_{k l}\left(\widetilde{\Gamma}^{3}\left(\partial_{k} u\right)\left(\partial_{l} u\right)-2\left(\partial_{k} u\right)\left(\partial_{l} \Gamma^{3} u\right)\right)
\end{aligned}
$$

can be estimated as follows

$$
\|R\|_{\mathbf{L}^{2}} \leq C\|\nabla \Gamma u\|_{\mathbf{L}^{4}}\left\|\nabla \Gamma^{2} u\right\|_{\mathbf{L}^{4}}+C\|\Gamma \Delta u\|_{\mathbf{L}^{4}}^{2}+C\|\nabla u\|_{\mathbf{W}_{\infty}^{1}}\left\|\Gamma^{3} u\right\|_{\mathbf{L}^{2}} .
$$

By Lemma 3.3 we have an optimal time decay estimate $\|\nabla u\|_{\mathbf{W}_{\infty}^{1}} \leq C \varepsilon t^{-1}$. By Lemma 3.2 we find $\|\Gamma \nabla u\|_{\mathbf{L}^{4}} \leq C \varepsilon t^{-\frac{1}{2}},\left\|\Gamma^{2} \nabla u\right\|_{\mathbf{L}^{4}} \leq C \varepsilon t^{\gamma-\frac{1}{2}}$ and $\|\Gamma \Delta u\|_{\mathbf{L}^{4}} \leq$ $C \varepsilon t^{\frac{\gamma}{2}-\frac{1}{2}}$. Therefore we obtain the estimate

$$
\|R\|_{\mathbf{L}^{2}} \leq C \varepsilon^{2} t^{\gamma-1}
$$

To treat the derivative loss in Eq. (3.2), we apply Lemma 2.2

$$
\begin{aligned}
& \frac{d}{d t}\|\mathcal{S} v\|_{\mathbf{L}^{2}}^{2}+2 t^{-1} \sum_{j=1}^{2}\left\|\left\langle\frac{x_{j}}{t}\right\rangle^{-1} \mathcal{S}\left|\partial_{j}\right|^{\frac{1}{2}} v\right\|_{\mathbf{L}^{2}}^{2} \\
& \leq C t^{-2}\|v\|_{\mathbf{L}^{2}}^{2}+C\|R\|_{\mathbf{L}^{2}}\|v\|_{\mathbf{L}^{2}}+C|(\mathcal{S N}(u, v), \mathcal{S} v)| .
\end{aligned}
$$

By Lemma 2.3 we find

$$
\begin{aligned}
|(\mathcal{S} v, \mathcal{S N}(u, v))| \leq & C \sum_{j=1,2}\left(\left\|\left\langle\frac{x}{t}\right\rangle^{2} \nabla u\right\|_{\mathbf{L}^{\infty}}+\left\|\left\langle\frac{x}{t}\right\rangle \nabla u\right\|_{\mathbf{W}_{\infty}^{1}}\right) \\
& \times\left(\left\|\left\langle\frac{x_{j}}{t}\right\rangle^{-1} \mathcal{S}\left|\partial_{j}\right|^{\frac{1}{2}} v\right\|_{\mathbf{L}^{2}}^{2}+\|v\|_{\mathbf{L}^{2}}^{2}\right) .
\end{aligned}
$$

Then by the condition of the lemma we get

$$
|(\mathcal{S} v, \mathcal{S N}(u, v))| \leq C t^{-1} \varepsilon \sum_{j=1,2}\left\|\left\langle\frac{x_{j}}{t}\right\rangle^{-1} \mathcal{S}\left|\partial_{j}\right|^{\frac{1}{2}} v\right\|_{\mathbf{L}^{2}}^{2}+C t^{-1} \varepsilon\|v\|_{\mathbf{L}^{2}}^{2} .
$$

Therefore we find

$$
\begin{aligned}
\frac{d}{d t}\|\mathcal{S} v\|_{\mathbf{L}^{2}}^{2} & \leq C t^{-1}\left(\varepsilon+t^{-1}\right)\|v\|_{\mathbf{L}^{2}}^{2}+(C \varepsilon-2) \sum_{j=1,2}\left\|\left\langle\frac{x_{j}}{t}\right\rangle^{-1} \mathcal{S}\left|\partial_{j}\right|^{\frac{1}{2}} v\right\|_{\mathbf{L}^{2}}^{2} \\
& \leq C t^{-1}\left(\varepsilon+t^{-1}\right)\|\mathcal{S} v\|_{\mathbf{L}^{2}}^{2}
\end{aligned}
$$

for $t \in[1, T]$. Integrating we get the estimate

$$
\left\|\Gamma^{3} u\right\|_{\mathbf{L}^{2}} \leq C\|\mathcal{S} v\|_{\mathbf{L}^{2}} \leq C t^{C \varepsilon}\left\|u_{0}\right\|_{\mathbf{H}^{6} \cap \mathbf{H}^{0,3}} .
$$

Lemma 3.4 is proved.

Next we estimate the norm $\left\|\Gamma^{2} u\right\|_{\mathbf{L}^{2}}$.

Lemma 3.5. Let the initial data $u_{0} \in \mathbf{H}^{6} \cap \mathbf{H}^{0,3}$. Assume that for the solutions of Eq. (1.1) the estimate $\|u\|_{\mathbf{X}_{T}} \leq C \varepsilon$ is true. Then the estimates are valid

$$
t^{\frac{4}{3}-2 \gamma}\left\|\mathcal{L} \Gamma^{2} h\right\|_{\mathbf{L}^{2}}+t^{1-5 \gamma}\|\mathcal{L} \mathcal{J} \Gamma h\|_{\mathbf{L}^{2}} \leq C \varepsilon^{2},
$$


and

$$
\left\|\Gamma^{2} u\right\|_{\mathbf{L}^{2}} \leq C\left\|u_{0}\right\|_{\mathbf{H}^{6} \cap \mathbf{H}^{0,3}}+C \varepsilon^{2}
$$

for all $t \in[1, T]$.

Proof. We apply the operator $\Gamma^{2}$ to Eq. (1.12)

$$
\begin{aligned}
& \mathcal{L} \Gamma^{2} h=\widetilde{\Gamma}^{2} a \mathcal{N}(\nabla u, \nabla u)+2 \widetilde{\Gamma}^{2} t B^{2} \mathcal{N}(\nabla u, \nabla \mathcal{L} u) \\
& \quad+\widetilde{\Gamma}^{2} \sum_{j=1,2} b_{j} \mathcal{N}\left(\nabla u, \mathcal{J}_{j} \nabla u\right)-\widetilde{\Gamma}^{2} \sum_{j=1,2} i B^{2} \mathcal{N}\left(\partial_{j} \nabla u, \mathcal{J}_{j} \nabla u\right),
\end{aligned}
$$

where $a=B^{2}+\mathcal{L}\left(t B^{2}\right)+2 i(x \cdot \nabla) B^{2}$ and $b_{j}=t^{-1} x_{j} B^{2}-2 i\left(\partial_{j} B^{2}\right)$. We next estimate the terms in Eq. (3.3). Since by a direct calculation we have $\left|\nabla B^{2}\right| \leq C t^{-\frac{1}{2}} B^{3},\left|\mathcal{L}\left(t B^{2}\right)\right| \leq C B^{2}$, we see that $|a|=\mid B^{2}+\mathcal{L}\left(t B^{2}\right)+2 i(x$. $\nabla) B^{2} \mid \leq C B^{2}$ and $\left|b_{j}\right|=\left|t^{-1} x_{j} B^{2}-2 i\left(\partial_{j} B^{2}\right)\right| \leq C t^{-\frac{1}{2}} B$. By Lemma 3.2 we have $\|B \nabla \Gamma u\|_{\mathbf{L}^{4}} \leq C \varepsilon t^{-\frac{3}{4}}$ and $\left\|B \nabla \Gamma^{2} u\right\|_{\mathbf{L}^{2}} \leq C \varepsilon t^{-\frac{1}{2}}$. Hence we have for the first summand in (3.3)

$$
\left\|\widetilde{\Gamma}^{2} a \mathcal{N}(\nabla u, \nabla u)\right\|_{\mathbf{L}^{2}} \leq C\|B \nabla \Gamma u\|_{\mathbf{L}^{4}}^{2}+C\|\nabla u\|_{\mathbf{W}_{\infty}^{1}}\left\|B^{2} \Gamma^{2} \nabla u\right\|_{\mathbf{L}^{2}} \leq C \varepsilon^{2} t^{-\frac{3}{2}} \text {. }
$$

Again by Lemma 3.2 we find $\left\|B \nabla \Gamma^{2} u\right\|_{\mathbf{L}^{p}} \leq C \varepsilon t^{\gamma+\frac{1}{p}-1},\|B \Delta u\|_{\mathbf{L}^{p}} \leq$ $C \varepsilon t^{\frac{2}{p}-\frac{3}{2}},\left\|B \Delta \Gamma^{2} u\right\|_{\mathbf{L}^{p}} \leq C \varepsilon t^{\gamma+\frac{2}{p}-\frac{3}{2}}$ and $\left\|\Gamma^{2} u\right\|_{\mathbf{L}^{p}} \leq C \varepsilon t^{\gamma+\frac{2}{p}-1}$. Thus we obtain for the second summand in (3.3)

$$
\begin{aligned}
& \left\|\widetilde{\Gamma}^{2} t B^{2} \mathcal{N}(\nabla u, \nabla \mathcal{L} u)\right\|_{\mathbf{L}^{2}} \leq C t\|\nabla u\|_{\mathbf{L}^{\infty}}\left\|B \nabla \Gamma^{2} u\right\|_{\mathbf{L}^{4}}\|B \Delta u\|_{\mathbf{L}^{4}} \\
& \quad+C t\|\nabla u\|_{\mathbf{L}^{\infty}}^{2}\left\|B^{2} \Delta \Gamma^{2} u\right\|_{\mathbf{L}^{2}}+C t\|\nabla \Gamma u\|_{\mathbf{L}^{6}}\|B \nabla \Gamma u\|_{\mathbf{L}^{6}}\|B \Delta u\|_{\mathbf{L}^{6}} \\
& \quad+C t\|\nabla u\|_{\mathbf{L}^{\infty}}\|B \nabla \Gamma u\|_{\mathbf{L}^{4}}\|B \Delta \Gamma u\|_{\mathbf{L}^{4}}+C t\left\|\Gamma^{2} u\right\|_{\mathbf{L}^{6}}\|B \Delta u\|_{\mathbf{L}^{6}}^{2} \leq C \varepsilon^{2} t^{3 \gamma-\frac{3}{2}}
\end{aligned}
$$

We use the Sobolev imbedding inequality $\|\mathcal{J} \nabla \Gamma u\|_{\mathbf{L}^{3}} \leq\|\mathcal{J} \nabla \Gamma u\|_{\mathbf{H}^{1}} \leq$ $\left\|\mathcal{J} \nabla \Gamma^{2} u\right\|_{\mathbf{L}^{2}} \leq C \varepsilon t^{\gamma}$. Then for the third summand in (3.3) we find

$$
\begin{aligned}
& \left\|\widetilde{\Gamma}^{2} \sum_{j=1,2} b_{j} \mathcal{N}\left(\nabla u, \mathcal{J}_{j} \nabla u\right)\right\|_{\mathbf{L}^{2}} \leq C t^{-\frac{1}{2}}\|\nabla u\|_{\mathbf{W}_{\infty}^{1}}\left\|\mathcal{J} \nabla \Gamma^{2} u\right\|_{\mathbf{L}^{2}} \\
& \quad+C t^{-\frac{1}{2}}\left\|B \nabla \Gamma^{2} u\right\|_{\mathbf{L}^{6}}\|\mathcal{J} \nabla \Gamma u\|_{\mathbf{L}^{3}} \leq C \varepsilon^{2} t^{\gamma-\frac{3}{2}}+C \varepsilon^{2} t^{2 \gamma-\frac{4}{3}} \leq C \varepsilon^{2} t^{2 \gamma-\frac{4}{3}} .
\end{aligned}
$$

Finally by Lemma 3.2 we get $\left\|B^{2} \Delta \Gamma u\right\|_{\mathbf{L}^{\infty}} \leq C\left\|B^{2} \Delta \Gamma^{2} u\right\|_{\mathbf{L}^{\frac{2}{\gamma}}} \leq C \varepsilon t^{2 \gamma-\frac{3}{2}}$, $\left\|\mathcal{J} \nabla \Gamma^{2} u\right\|_{\mathbf{L}^{2}} \leq C \varepsilon t^{\gamma}$. Therefore we estimate the last summand in (3.3)

$$
\begin{aligned}
\left\|\widetilde{\Gamma}^{2} \sum_{j=1,2} B^{2} \mathcal{N}\left(\partial_{j} \nabla u, \mathcal{J}_{j} \nabla u\right)\right\|_{\mathbf{L}^{2}} \leq & C\left\|B^{2} \Delta \Gamma u\right\|_{\mathbf{L}^{\infty}}\left\|\mathcal{J} \nabla \Gamma^{2} u\right\|_{\mathbf{L}^{2}} \\
& +C\left\|B^{2} \Delta \Gamma^{2} u\right\|_{\mathbf{L}^{\frac{2}{\gamma}}}\left\|\mathcal{J} \nabla \Gamma^{2} u\right\|_{\mathbf{L}^{2}} \leq C \varepsilon^{2} t^{3 \gamma-\frac{3}{2}}
\end{aligned}
$$

From (3.3) we obtain $\left\|\mathcal{L} \Gamma^{2} h\right\|_{\mathbf{L}^{2}} \leq C \varepsilon^{2} t^{2 \gamma-\frac{4}{3}}$. Then

$$
\begin{aligned}
\frac{d}{d t}\left\|\Gamma^{2} h\right\|_{\mathbf{L}^{2}}^{2} & =2 \operatorname{Im}\left(\Gamma^{2} h, \mathcal{L} \Gamma^{2} h\right) \leq 2\left\|\Gamma^{2} h\right\|_{\mathbf{L}^{2}}\left\|\mathcal{L} \Gamma^{2} h\right\|_{\mathbf{L}^{2}} \\
& \leq C \varepsilon^{2} t^{2 \gamma-\frac{4}{3}}\left\|\Gamma^{2} h\right\|_{\mathbf{L}^{2}} .
\end{aligned}
$$


Hence integrating we find

$$
\left\|\Gamma^{2} h\right\|_{\mathbf{L}^{2}} \leq C\left\|u_{0}\right\|_{\mathbf{H}^{6} \cap \mathbf{H}^{0,3}}+C \varepsilon^{2}
$$

for $t \in[1, T]$. Since by Lemma $3.2\|\nabla \Gamma u\|_{\mathbf{L}^{\infty}} \leq C\|\nabla \Gamma u\|_{\mathbf{W}_{2 / \gamma}^{1}} \leq C \varepsilon t^{2 \gamma-1}$ and $\left\|B \nabla \Gamma^{2} u\right\|_{\mathbf{L}^{2}} \leq C \varepsilon t^{-\frac{1}{2}}$ we get

$$
\left\|\Gamma^{2} t B^{2} \mathcal{N}(\nabla u, \nabla u)\right\|_{\mathbf{L}^{2}} \leq C t\|\nabla \Gamma u\|_{\mathbf{L}^{\infty}}\left\|B \nabla \Gamma^{2} u\right\|_{\mathbf{L}^{2}} \leq C \varepsilon^{2} t^{2 \gamma-\frac{1}{2}} .
$$

Hence

$$
\left\|\Gamma^{2} u\right\|_{\mathbf{L}^{2}} \leq\left\|\Gamma^{2} h\right\|_{\mathbf{L}^{2}}+\left\|\Gamma^{2} t B^{2} \mathcal{N}(\nabla u, \nabla u)\right\|_{\mathbf{L}^{2}} \leq C\left\|u_{0}\right\|_{\mathbf{H}^{6} \cap \mathbf{H}^{0,3}}+C \varepsilon^{2} .
$$

Next we apply the operator $\mathcal{J} \Gamma$ to Eq. (1.12)

$$
\begin{aligned}
\mathcal{L} \mathcal{J} \Gamma h= & \mathcal{J} \Gamma a \mathcal{N}(\nabla u, \nabla u)+2 \mathcal{J} \Gamma t B^{2} \mathcal{N}(\nabla u, \nabla \mathcal{L} u) \\
& +\mathcal{J} \Gamma \sum_{j=1,2} b_{j} \mathcal{N}\left(\nabla u, \mathcal{J}_{j} \nabla u\right)-\mathcal{J} \Gamma \sum_{j=1,2} i B^{2} \mathcal{N}\left(\partial_{j} \nabla u, \mathcal{J}_{j} \nabla u\right) .
\end{aligned}
$$

By Lemma 3.2 we have $\|\nabla \Gamma u\|_{\mathbf{L}^{\infty}} \leq C \varepsilon t^{2 \gamma-1},\|\mathcal{J} \nabla \Gamma u\|_{\mathbf{L}^{2}} \leq C \varepsilon$ and $\|B \nabla \Gamma u\|_{\mathbf{L}^{2}} \leq C \varepsilon t^{\gamma-\frac{1}{2}}$. Hence we find for the first summand in (3.4)

$$
\begin{aligned}
\|\mathcal{J} \Gamma a \mathcal{N}(\nabla u, \nabla u)\|_{\mathbf{L}^{2}} & \leq C\|\nabla \Gamma u\|_{\mathbf{L}^{\infty}}\left(\|\mathcal{J} \nabla \Gamma u\|_{\mathbf{L}^{2}}+C t^{\frac{1}{2}}\|B \nabla \Gamma u\|_{\mathbf{L}^{2}}\right) \\
& \leq C \varepsilon^{2} t^{3 \gamma-1} .
\end{aligned}
$$

Again by Lemma 3.2 we get $\|B \Delta \Gamma u\|_{\mathbf{L}^{\frac{2}{\gamma}}} \leq C \varepsilon t^{2 \gamma-\frac{3}{2}},\|B \Delta \Gamma u\|_{\mathbf{L}^{2}} \leq$ $C \varepsilon t^{\gamma-\frac{1}{2}},\|B \mathcal{J} \Delta \Gamma u\|_{\mathbf{L}^{2}} \leq C \varepsilon t^{-\frac{1}{2}}$, and $\|\mathcal{J} \nabla u\|_{\mathbf{H}^{1}} \leq C \varepsilon$. Thus we obtain for the second summand in (3.4)

$$
\begin{aligned}
\left\|\mathcal{J} \Gamma t B^{2} \mathcal{N}(\nabla u, \nabla \mathcal{L} u)\right\|_{\mathbf{L}^{2}} \leq & C t\|\nabla \Gamma u\|_{\mathbf{L}^{\infty}}\|B \Delta \Gamma u\|_{\mathbf{L}^{\frac{2}{\gamma}}}\|\mathcal{J} \nabla \Gamma u\|_{\mathbf{L}^{2}} \\
& +C t\|\nabla \Gamma u\|_{\mathbf{L}^{\infty}}^{2}\|B \mathcal{J} \Delta \Gamma u\|_{\mathbf{L}^{2}} \\
& +C t^{\frac{3}{2}}\|\nabla \Gamma u\|_{\mathbf{L}^{\infty}}^{2}\|B \Delta \Gamma u\|_{\mathbf{L}^{2}} \leq C \varepsilon^{2} t^{5 \gamma-1} .
\end{aligned}
$$

By Lemma 3.2 we find $\left\|B \mathcal{J}^{2} \nabla \Gamma u\right\|_{\mathbf{L}^{2}} \leq C \varepsilon t^{\frac{1}{2}},\|B \mathcal{J} \nabla u\|_{\mathbf{L}^{\frac{1}{\gamma}}} \leq C \varepsilon t^{\gamma-\frac{1}{2}}$, $\|\mathcal{J} \nabla \Gamma u\|_{\mathbf{L}^{2}} \leq C \varepsilon$, then

$$
\begin{aligned}
\left\|\mathcal{J} \Gamma \sum_{j=1,2} b_{j} \mathcal{N}\left(\nabla u, \mathcal{J}_{j} \nabla u\right)\right\|_{\mathbf{L}^{2}} \leq & C t^{-\frac{1}{2}}\|\nabla \Gamma u\|_{\mathbf{L}^{\infty}}\left\|B \mathcal{J}^{2} \nabla \Gamma u\right\|_{\mathbf{L}^{2}} \\
& +C t^{-\frac{1}{2}}\|B \mathcal{J} \nabla u\|_{\mathbf{L}^{\frac{1}{\gamma}}}\|\mathcal{J} \nabla \Gamma u\|_{\mathbf{L}^{2}} \\
& +C\|\nabla u\|_{\mathbf{L}^{\infty}}\|\mathcal{J} \nabla u\|_{\mathbf{L}^{2}} \leq C \varepsilon^{2} t^{2 \gamma-1} .
\end{aligned}
$$

Finally by Lemma 3.2 we obtain $\|B \Delta \Gamma u\|_{\mathbf{L}} \frac{2}{\gamma} \leq C \varepsilon t^{2 \gamma-\frac{3}{2}},\|B \mathcal{J} \Delta \Gamma u\|_{\mathbf{L}^{2}} \leq$ $C \varepsilon t^{-\frac{1}{2}},\|B \mathcal{J} \nabla \Gamma u\|_{\mathbf{L}^{\frac{1}{\gamma}}} \leq C \varepsilon t^{\gamma-\frac{1}{2}}$. Therefore we estimate the last summand in $(3.4)$ 


$$
\begin{aligned}
\left\|\mathcal{J} \Gamma \sum_{j=1,2} B^{2} \mathcal{N}\left(\partial_{j} \nabla u, \mathcal{J}_{j} \nabla u\right)\right\|_{\mathbf{L}^{2}} \leq & C\|B \Delta \Gamma u\|_{\mathbf{L}^{\frac{2}{\gamma}}}\left\|B \mathcal{J}^{2} \nabla \Gamma u\right\|_{\mathbf{L}^{2}} \\
& +C\|B \mathcal{J} \nabla \Gamma u\|_{\mathbf{L}^{\frac{1}{\gamma}}}\|B \mathcal{J} \Delta \Gamma u\|_{\mathbf{L}^{2}} \\
& +C \sqrt{t}\|B \Delta \Gamma u\|_{\mathbf{L}^{\frac{2}{\gamma}}}\|\mathcal{J} \nabla \Gamma u\|_{\mathbf{L}^{2}} \leq C \varepsilon^{2} t^{2 \gamma-1} .
\end{aligned}
$$

Thus from (3.4) we get the estimate

$$
\|\mathcal{L} \mathcal{J} \Gamma h\|_{\mathbf{L}^{2}} \leq C \varepsilon^{2} t^{5 \gamma-1} .
$$

Lemma 3.5 is proved.

In the next lemma we estimate the norm $\left\|\mathcal{J} \Gamma^{2} u\right\|_{\mathbf{L}^{2}}$.

Lemma 3.6. Let the initial data $u_{0} \in \mathbf{H}^{6} \cap \mathbf{H}^{0,3}$. Assume that for the solutions of Eq. (1.1) the estimate $\|u\|_{\mathbf{x}_{T}} \leq C \varepsilon$ is true. Then the estimate is valid

$$
\left\|\mathcal{J} \Gamma^{2} u\right\|_{\mathbf{L}^{2}} \leq C\left\|u_{0}\right\|_{\mathbf{H}^{6} \cap \mathbf{H}^{0,3}}+C \varepsilon^{2} t^{3 \gamma}
$$

for $t \in[1, T]$.

Proof. We apply the operator $\mathcal{J}$ to Eq. (1.1) to obtain

$$
\mathcal{L} \mathcal{J} u=2 \mathcal{N}(\nabla u, \mathcal{J} \nabla u)-x \mathcal{N}(\nabla u, \nabla u) .
$$

To exclude the last term from Equation (3.5) as in the derivation of Equation (1.12) we use identity (1.10). We express the derivative $\nabla=i t^{-1} x-i t^{-1} \mathcal{J}$, then we find the identity

$$
\begin{aligned}
\mathcal{L}(A v w)= & \left(-t^{-2}|x|^{2} A+\mathcal{L} A+2 i t^{-1}(x \cdot \nabla) A\right) v w+A w \mathcal{L} v+A v \mathcal{L} w \\
& +t^{-2} w(x A-i t \nabla A) \cdot \mathcal{J} v+t^{-2} v(x A-i t \nabla A) \cdot \mathcal{J} w-t^{-2} A \mathcal{J} w \cdot \mathcal{J} v .
\end{aligned}
$$

We apply the identity (3.6) with $A_{k l}=\lambda_{k l} x t B^{2}, v=\partial_{k} u, w=\partial_{l} u$, then for the new dependent variable $g=\mathcal{J} u-x t B^{2} \mathcal{N}(\nabla u, \nabla u)$ we find

$$
\begin{aligned}
\mathcal{L} g= & 2 \mathcal{N}(\nabla u, \mathcal{J} \nabla u)-a \mathcal{N}(\nabla u, \nabla u)-2 x t B^{2} \mathcal{N}(\nabla u, \nabla \mathcal{L} u) \\
& +\sum_{j=1,2}\left(b_{j} \mathcal{N}\left(\nabla u, \mathcal{J}_{j} \nabla u\right)+t^{-1} x B^{2} \mathcal{N}\left(\mathcal{J}_{j} \nabla u, \mathcal{J}_{j} \nabla u\right)\right)
\end{aligned}
$$

where $a=x B^{2}+\mathcal{L}\left(x t B^{2}\right)+2 i \sum_{j=1,2} x_{j} \partial_{j}\left(x B^{2}\right), b_{j}=2 i \partial_{j}\left(x B^{2}\right)-2 t^{-1} x_{j} x B^{2}$. We now apply the operator $\Gamma^{2}$ to get

$$
\begin{aligned}
\mathcal{L} \Gamma^{2} g= & 2 \widetilde{\Gamma}^{2} \mathcal{N}(\nabla u, \mathcal{J} \nabla u)-\widetilde{\Gamma}^{2} a \mathcal{N}(\nabla u, \nabla u)-2 \widetilde{\Gamma}^{2} x t B^{2} \mathcal{N}(\nabla u, \nabla \mathcal{L} u) \\
& +\sum_{j=1,2}\left(\widetilde{\Gamma}^{2} b_{j} \mathcal{N}\left(\nabla u, \mathcal{J}_{j} \nabla u\right)+i \widetilde{\Gamma}^{2} x B^{2} \mathcal{N}\left(\partial_{j} \nabla u, \mathcal{J}_{j} \nabla u\right)\right) .
\end{aligned}
$$

Note that $|a| \leq C \sqrt{t} B$ and $\left|b_{j}\right| \leq C$. We next estimate the terms in Equation (3.7). By Lemma 3.2 we have $\left\|\nabla \Gamma^{2} u\right\|_{\mathbf{L}^{p}} \leq C \varepsilon t^{\gamma+\frac{2}{p}-1},\|\mathcal{J} \nabla \Gamma u\|_{\mathbf{L}^{p}}+\|\mathcal{J} \Delta \Gamma u\|_{\mathbf{L}^{p}}$ 
$\leq\left\|\mathcal{J} \nabla \Gamma^{2} u\right\|_{\mathbf{L}^{2}} \leq C \varepsilon t^{\gamma}$. Therefore by the Hölder inequality

$$
\begin{aligned}
& \left\|\widetilde{\Gamma}^{2} \mathcal{N}(\nabla u, \mathcal{J} \nabla u)\right\|_{\mathbf{L}^{2}}+\left\|\sum_{j=1,2} \widetilde{\Gamma}^{2} b_{j} \mathcal{N}\left(\nabla u, \mathcal{J}_{j} \nabla u\right)\right\|_{\mathbf{L}^{2}} \\
& \leq C\left\|\nabla \Gamma^{2} u\right\|_{\mathbf{L}^{\frac{2}{\gamma}}}\left(\|\mathcal{J} \nabla \Gamma u\|_{\mathbf{L}^{\frac{2}{1-\gamma}}}+\|\mathcal{J} \Delta \Gamma u\|_{\mathbf{L}^{\frac{2}{1-\gamma}}}\right) \\
& \quad+C\|\nabla u\|_{\mathbf{W}_{\infty}^{1}}\left\|\mathcal{J} \nabla \Gamma^{2} u\right\|_{\mathbf{L}^{2}} \leq C \varepsilon^{2} t^{3 \gamma-1} .
\end{aligned}
$$

By Lemma 3.2 we have $\|\nabla \Gamma u\|_{\mathbf{L}^{\infty}} \leq C\|\nabla \Gamma u\|_{\mathbf{W}_{2 / \gamma}^{1}} \leq C \varepsilon t^{2 \gamma-1}$ and $\left\|B \nabla \Gamma^{2} u\right\|_{\mathbf{H}^{1}} \leq C \varepsilon t^{\gamma-\frac{1}{2}}$. Then we estimate the second term in Eq. (3.7)

$\left\|\widetilde{\Gamma}^{2} a \mathcal{N}(\nabla u, \nabla u)\right\|_{\mathbf{L}^{2}} \leq C \sqrt{t}\left(\|\nabla \Gamma u\|_{\mathbf{L}^{\infty}}+\|\nabla u\|_{\mathbf{W}_{\infty}^{1}}\right)\left\|B \nabla \Gamma^{2} u\right\|_{\mathbf{L}^{2}} \leq C \varepsilon^{2} t^{2 \gamma-1}$

and similarly the third term in Eq. (3.7)

$\left\|\widetilde{\Gamma}^{2} x t B^{2} \mathcal{N}(\nabla u, \nabla \mathcal{L} u)\right\|_{\mathbf{L}^{2}} \leq C t^{\frac{3}{2}}\|\nabla u\|_{\mathbf{W}_{\infty}^{1}}\|\nabla \Gamma u\|_{\mathbf{L}^{\infty}}\left\|B \nabla \Gamma^{2} u\right\|_{\mathbf{H}^{1}} \leq C \varepsilon^{3} t^{2 \gamma-1}$.

Finally by Lemma 3.2 we find

$$
\begin{aligned}
\|B \mathcal{J} \nabla \Gamma u\|_{\mathbf{L}^{\infty}} & \leq C\|x B \nabla \Gamma u\|_{\mathbf{L}^{\infty}}+C t\|B \Delta \Gamma u\|_{\mathbf{L}^{\infty}} \\
& \leq C t^{\frac{1}{2}}\left\|\nabla \Gamma^{2} u\right\|_{\mathbf{L}^{\frac{2}{\gamma}}}+C t\left\|B \Delta \Gamma^{2} u\right\|_{\mathbf{L}^{\frac{2}{\gamma}}} \leq C \varepsilon t^{2 \gamma-\frac{1}{2}},
\end{aligned}
$$

and $\left\|\mathcal{J} \nabla \Gamma^{2} u\right\|_{\mathbf{L}^{2}} \leq C \varepsilon t^{\gamma}$. Therefore we estimate the last term in equation (3.7)

$$
\begin{aligned}
& \left\|\sum_{j=1,2} \widetilde{\Gamma}^{2} t^{-1} x B^{2} \mathcal{N}\left(\mathcal{J}_{j} \nabla u, \mathcal{J}_{j} \nabla u\right)\right\|_{\mathbf{L}^{2}} \\
& \quad \leq C t^{-\frac{1}{2}}\|B \mathcal{J} \nabla \Gamma u\|_{\mathbf{L}^{\infty}}\left\|\mathcal{J} \nabla \Gamma^{2} u\right\|_{\mathbf{L}^{2}} \leq C \varepsilon^{3} t^{3 \gamma-1} .
\end{aligned}
$$

Therefore we find from Eq. (3.7)

$$
\frac{d}{d t}\left\|\Gamma^{2} g\right\|_{\mathbf{L}^{2}}^{2} \leq 2\left\|\Gamma^{2} g\right\|_{\mathbf{L}^{2}}\left\|\mathcal{L} \Gamma^{2} g\right\|_{\mathbf{L}^{2}} \leq C \varepsilon^{2} t^{3 \gamma-1}\left\|\Gamma^{2} g\right\|_{\mathbf{L}^{2}} .
$$

Integration with respect to $t$ yields

$$
\left\|\Gamma^{2} g\right\|_{\mathbf{L}^{2}} \leq C\left\|u_{0}\right\|_{\mathbf{H}^{6} \cap \mathbf{H}^{0,3}}+C \varepsilon^{2} t^{3 \gamma}
$$

for $t \in[1, T]$. Since by Lemma $3.2\|\nabla \Gamma u\|_{\mathbf{L}^{\infty}} \leq C \varepsilon t^{\gamma-1}$ and $\left\|B \nabla \Gamma^{2} u\right\|_{\mathbf{L}^{2}} \leq$ $C \varepsilon t^{\gamma-\frac{1}{2}}$, we get

$$
\left\|\Gamma^{2} x t B^{2} \mathcal{N}(\nabla u, \nabla u)\right\|_{\mathbf{L}^{2}} \leq C t^{\frac{3}{2}}\|\nabla \Gamma u\|_{\mathbf{L}^{\infty}}\left\|B \nabla \Gamma^{2} u\right\|_{\mathbf{L}^{2}} \leq C \varepsilon^{2} t^{2 \gamma} .
$$

Thus the estimate of the lemma follows

$$
\begin{aligned}
\left\|\mathcal{J} \Gamma^{2} u\right\|_{\mathbf{L}^{2}} & \leq\left\|\Gamma^{2} g\right\|_{\mathbf{L}^{2}}+\left\|\Gamma^{2} x t B^{2} \mathcal{N}(\nabla u, \nabla u)\right\|_{\mathbf{L}^{2}} \\
& \leq C\left\|u_{0}\right\|_{\mathbf{H}^{6} \cap \mathbf{H}^{0,3}}+C \varepsilon^{2} t^{3 \gamma} .
\end{aligned}
$$

Lemma 3.6 is proved. 


\section{Proof of Theorem 1.1}

First we state the local existence result (see [1]).

Theorem 4.1. Assume that the initial data $u_{0} \in \mathbf{H}^{6} \cap \mathbf{H}^{0,3}$ are small. Then for some time $T>1$ there exists a unique solution $u \in \mathbf{C}\left([1, T] ; \mathbf{H}^{6} \cap \mathbf{H}^{0,3}\right)$ of the Cauchy problem (1.1).

We define the functional space

$$
\mathbf{X}_{T}=\left\{u \in \mathbf{C}\left([1, T] ; \mathbf{L}^{2}\right) ;\|u\|_{\mathbf{X}_{T}}<\infty\right\}
$$

where the norm

$$
\|u\|_{\mathbf{X}_{T}}=\sup _{t \in[1, T]}\left(t^{-\gamma}\left\|\Gamma^{3} u\right\|_{\mathbf{L}^{2}}+t^{-3 \gamma}\left\|\mathcal{J} \Gamma^{2} u\right\|_{\mathbf{L}^{2}}+\left\|\Gamma^{2} u\right\|_{\mathbf{L}^{2}}\right),
$$

and $\Gamma=(1, \mathcal{I}, \Omega, \Delta), \mathcal{I}=(x \cdot \nabla)-2 t \partial_{t}, \Omega=x_{2} \partial_{1}-x_{1} \partial_{2}$, with some small $\gamma>0$.

Let us prove a priori estimate

$$
\|u\|_{\mathbf{X}_{T}}<C \varepsilon
$$

for all $T>1$. On the contrary suppose that estimate (4.1) is not fulfilled for all $T>1$. Then by the continuity there exists the first time $1<\widetilde{T}<\infty$, such that

$$
\|u\|_{\mathbf{X}_{\widetilde{T}}}=C \varepsilon .
$$

So we can apply successively Lemmas 3.4 - 3.6 to find estimates for the norm

$$
\begin{aligned}
\|u\|_{\mathbf{X}_{\widetilde{T}}} & =\sup _{t \in[1, \widetilde{T}]}\left(t^{-\gamma}\left\|\Gamma^{3} u\right\|_{\mathbf{L}^{2}}+\left\|\Gamma^{2} u\right\|_{\mathbf{L}^{2}}+t^{-3 \gamma}\left\|\mathcal{J} \Gamma^{2} u\right\|_{\mathbf{L}^{2}}\right) \\
& \leq C\left\|u_{0}\right\|_{\mathbf{H}^{6} \cap \mathbf{H}^{0,3}}+C \varepsilon^{2}<C \varepsilon
\end{aligned}
$$

provided that the norm $\left\|u_{0}\right\|_{\mathbf{H}^{6} \cap \mathbf{H}^{0,3}}$ and $\varepsilon>0$ are sufficiently small. This contradicts to (4.2). Therefore estimate (4.1) is true for all $T>1$. This proves the existence of a unique global solution $u \in \mathbf{C}\left(\mathbf{R}, \mathbf{H}^{6} \cap \mathbf{H}^{3,3}\right)$ to the Cauchy problem (1.1).

Finally let us prove the existence of the usual scattering states. Denote $\varphi(t)=\mathcal{F U}(-t) \nabla h(t)$. We apply operator $\mathcal{F U}(-t)$ to Eq. (1.12), then we get

$$
i \varphi_{t}=\mathcal{F U}(-t) R_{0}(t)
$$

for all $t \geq 1$, where

$$
\begin{aligned}
R_{0}(t)= & \nabla a \mathcal{N}(\nabla u, \nabla u)+2 t \mathcal{F U}(-t) \nabla B^{2} \mathcal{N}(\nabla u, \nabla \mathcal{L} u) \\
& +\mathcal{F U}(-t) \nabla \sum_{j=1,2} b_{j} \mathcal{N}\left(\nabla u, \mathcal{J}_{j} \nabla u\right)-\mathcal{F U}(-t) \nabla \sum_{j=1,2} i B^{2} \mathcal{N}\left(\partial_{j} \nabla u, \mathcal{J}_{j} \nabla u\right) .
\end{aligned}
$$

By the estimate of Lemma 3.5 we find $\left\|\mathcal{F U}(-t) R_{0}(t)\right\|_{\mathbf{L}^{2}}=\left\|R_{0}(t)\right\|_{\mathbf{L}^{2}} \leq$ $\left\|\mathcal{L} \Gamma^{2} h\right\|_{\mathbf{L}^{2}} \leq C \varepsilon^{2} t^{2 \gamma-\frac{4}{3}}$. Therefore integrating Eq. (4.3) with respect to $t$, we obtain

$$
\|\varphi(t)-\varphi(s)\|_{\mathbf{L}^{2}} \leq C \int_{s}^{t}\left\|\mathcal{F U}(-\tau) R_{0}(\tau)\right\|_{\mathbf{L}^{2}} d \tau \leq C \varepsilon^{2} s^{2 \gamma-\frac{1}{3}}
$$


for $t>s>0$. Since $\nabla h=\nabla u+\nabla t B^{2} \mathcal{N}(\nabla u, \nabla u)$ and

$$
\begin{aligned}
& \left\|\nabla t B^{2} \mathcal{N}(\nabla u, \nabla u)\right\|_{\mathbf{L}^{2}} \leq C t\|\nabla u\|_{\mathbf{W}_{\infty}^{1}}\left\|B^{2} \nabla u\right\|_{\mathbf{H}^{1}} \\
& \quad \leq C\|\nabla u\|_{\mathbf{W}_{\infty}^{1}}\left(\left\|B^{2} x u\right\|_{\mathbf{H}^{1}}+\|\mathcal{J} u\|_{\mathbf{H}^{1}}\right) \leq C \varepsilon^{2} t^{3 \gamma-\frac{1}{2}},
\end{aligned}
$$

we find from inequality (4.4)

$$
\|\mathcal{F U}(-t) \nabla u(t)-\mathcal{F U}(-s) \nabla u(s)\|_{\mathbf{L}^{2}} \leq C \varepsilon^{2} s^{2 \gamma-\frac{1}{3}}
$$

for $t>s>0$. Hence there exists a unique final state $u_{+} \in \mathbf{L}^{2}$ such that $\left\|\nabla u(t)-\mathcal{U}(t) u_{+}\right\|_{\mathbf{L}^{2}} \leq C \varepsilon^{2} t^{2 \gamma-\frac{1}{3}}$ as $t \rightarrow \infty$. This completes the proof of Theorem 1.1.

\section{Acknowledgments}

We are very grateful to unknown referees for many useful suggestions and comments. The work of P.I.N. is partially supported by CONACYT and the work of N.H. is partially supported by KAKENHI (no. 19340030).

\section{References}

[1] Cazenave, Th.: Semilinear Schrödinger equations, Courant Institute of Mathematical Sciences, New York. pp. xiv+323 American Mathematical Society, Providence, RI (2003)

[2] Chang, N.-H., Shatah, J., Uhlenbeck, K.: Schrödinger maps. Commun. Pure Appl. Math. 53, 590-602 (2003)

[3] Chihara, H.: Global existence of small solutions to semilinear Schrödinger equations with gauge invariance. Publ. RIMS 31(5), 731-753 (1995)

[4] Chihara, H.: The initial value problem for cubic semilinear Schrödinger equations. Publ. RIMS 32(3), 445-471 (1996)

[5] Cohn, S.: Resonance and long time existence for the quadratic semilinear Schrödinger equation. Commun. Pure Appl. Math. 45, 973-1001 (1992)

[6] Cohn, S.: Global existence for the nonresonant Schrödinger equation in two space dimensions. Canad. Appl. Math. Q. 2, 247-282 (1994)

[7] Delort, J.-M.: Global solutions for small nonlinear long range perturbations of two dimensional Schrödinger equations. Mem. Soc. Math. Fr. 91, 94 (2002)

[8] Doi, S.: The initial value problem for the Schrödinger type equations and regularity of solutions. J. Math. Kyoto Univ. 34, 319-328 (1994)

[9] Germain, P., Masmoudi, N. and Shatah, J.: Global solutions for 3D quadratic Schrödinger equations. Int. Math. Res. Not. 3, 414-432 (2009)

[10] Ginibre, J.: Introduction aux équations de Schrödinger non linéaires (Paris Onze Edition). pp. L161 Université Paris-Sud, France (1998) 
[11] Ginibre, J., Hayashi, N.: Almost global existence of small solutions to quadratic nonlinear Schrödinger equations in three space dimensions. Math. Z 219, 119 $140(1995)$

[12] Hayashi, N.: Global existence of small solutions to quadratic nonlinear Schrödinger equations. Commun. Partial Diff. Equ. 18, 1109-1124 (1993)

[13] Hayashi, N.: Global and almost global solutions to quadratic nonlinear Schrödinger equations with small initial data. Dyn. Cont. Discrete Impuls. Syst. 2, 109-129 (1996)

[14] Hayashi, N., Hirata, H.: Local existence in time of small solutions to the elliptichyperbolic Davey-Stewartson system in the usual Sobolev space. Proc. Edinburgh Math. Soc. 40, 563-581 (1997)

[15] Hayashi, N., Hirata, H.: Global existence of small solutions to nonlinear Schrödinger equations. Nonlinear Anal. TMA 31, 671-685 (1998)

[16] Hayashi, N., Kaikina, E.I.: Local existence of solutions to the Cauchy problem for nonlinear Schrödinger equations. SUT J. Math. 34, 111-137 (1998)

[17] Hayashi, N., Kato, K.: Global existence of small analytic solutions to Schrödinger equations with quadratic nonlinearity. Commun. Partial Diff. Equ. 22, 773$798(1997)$

[18] Hayashi, N., Naumkin, P.I.: On the quadratic nonlinear Schrödinger equation in three space dimensions. Int. Math. Res. Not. 3, 115-132 (2000)

[19] Hayashi, N., Naumkin, P.I.: Global existence of small solutions to the quadratic nonlinear Schrödinger equations in two space dimensions. SIAM J. Math. Anal 32(6), 1390-1403 (2001)

[20] Hayashi, N., Miao, C., Naumkin, P.I.: Global existence of small solutions to the generalized derivative nonlinear Schrödinger equation. Asymptot. Anal. 21, 133147 (1999)

[21] Hayashi, N., Ozawa, T.: Remarks on nonlinear Schrö dinger equations in one space dimension. Diff. Integral Equ. 7, 453-461 (1994)

[22] Hayashi, N., Ozawa, T.: Global, small radially symmetric solutions to nonlinear Schrödinger equations and a gauge transformation. Diff. Integral Equ. 8, 10611072 (1995)

[23] Kato, J.: Existence and uniqueness of the solution to the modified Schrödinger map. Math. Res. Lett. 12(2-3), 171-186 (2005)

[24] Kato, T. : Nonlinear Schrödinger equations. In: Holden, H., Jensen, A. (eds.) Schrödinger Operators, Lecture Notes in Physics, vol. 345, pp. 218263. Springer, Berlin (1989)

[25] Kenig, C., Pollack, D., Staffilani, G. and Toro, T.: The Cauchy problem for Schrödinger flows into Kähler manifolds (29 Nov 2005). arXiv: math.AP/0511701 v1 
[26] Kenig, C., Ponce, G., Vega, L.: Small solutions to nonlinear Schrödinger equations. Ann. Inst. H. Poincaré Anal. Non Linéaire 10(3), 255-288 (1993)

[27] Kenig, C., Ponce, G., Vega, L.: Well-posedness and scattering results for the generalized Korteweg-de Vries equation via contraction principle. Commun. Pure Appl. Math. 46, 527-620 (1993)

[28] Kenig, C., Ponce, G., Vega, L.: Smoothing effects and local existence theory for the generalized nonlinear Schrödinger equations. Invent. Math. 134(3), 489545 (1998)

[29] Klainerman, S.: Weighted $\mathbf{L}^{\infty}$ and $\mathbf{L}^{1}$ estimates for solutions to the classical wave equation in three space dimensions. Commun. Pure Appl. Math. 37(2), 269$288(1984)$

[30] Klainerman, S.: Long-time behavior of solutions to nonlinear evolution equations. Arch. Rat. Mech. Anal. 78, 73-98 (1982)

[31] Klainerman, S., Ponce, G.: Global, small amplitude solutions to nonlinear evolution equations. Commun. Pure Appl. Math. 36, 133-141 (1983)

[32] Nahmod, A., Stefanov, A., Uhlenbeck, K.: On Schrödinger maps. Commun. Pure Appl. Math. 56(1), 114-151 (2003)

[33] Ozawa, T.: Remarks on quadratic nonlinear Schrödinger equations. Funkcial. Ekvac. 38, 217-232 (1995)

[34] Ozawa, T.: Finite energy solutions for the Schrödinger equations with quadratic nonlinearity in one space dimension. Funkcial. Ekvac. 41(3), 451-468 (1998)

[35] Ozawa, T., Zhai, J.: Global existence of small classical solutions to nonlinear Schrödinger equations. Ann. Inst. H. Poincaré Anal. Non Linéaire 25(2), 303311 (2008)

[36] Soyeur, A.: The Cauchy problem for the Ishimori equations. J. Funct. Anal. 105(2), 233-255 (1992)

[37] Sulem, C., Sulem, P.-L.: The Nonlinear Schrödinger Equation, Self-Focusing and Wave Collapse, Applied Mathematical Sciences, vol. 139. Springer, New York (1999)

[38] Shatah, J.: Global existence of small solutions to nonlinear evolution equations. J. Diff. Equ. 46, 409-425 (1982)

[39] Shatah, J.: Normal forms and quadratic nonlinear Klein-Gordon equations. Commun. Pure Appl. Math. 38, 685-696 (1985)

[40] Shatah, J., Zeng, C.: Schrödinger maps and anti-ferromagnetic chains. Commun. Math. Phys. 262(2), 299-315 (2006)

[41] Stein, E.M.: Singular Integrals and Differentiability Properties of Functions, vol. 30. Princeton University Press, Princeton, NJ (1970)

[42] Strauss, W.A.: Nonlinear scattering theory at low energy. J. Funct. Anal. 41, 110-133 (1981) 
[43] Tonegawa, S.: Global existence for a class of cubic nonlinear Schrödinger equations in one space dimension. Hokkaido Math. J. 30(2), 451-473 (2001)

Fernando Bernal-Vílchis, Pavel I. Naumkin

Instituto de Matemáticas,

UNAM Campus Morelia,

AP 61-3 (Xangari),

CP 58089 Morelia,

Michoacán,

Mexico

e-mail: fbernal@matmor.unam.mx

Pavel I. Naumkin

e-mail: pavelni@matmor.unam.mx

Nakao Hayashi

Department of Mathematics,

Graduate School of Science,

Osaka University,

Osaka,

Tokyonaka,

Japan

e-mail: nhayashi@math.sci.osaka-u.ac.jp

Received: 7 May 2010.

Accepted: 6 January 2011. 